\title{
Transport properties of heavy particles in high Reynolds number turbulence
}

\author{
Piero Olla \\ ISAC-CNR \\ Sezione di Lecce \\ 73100 Lecce Italy
}

\begin{abstract}
The statistical properties of heavy particle trajectories in high Reynolds numbers turbulent flows are analyzed. Dimensional analysis assuming Kolmogorov scaling is compared with the result of numerical simulation using a synthetic turbulence advecting field. The non-Markovian nature of the fluid velocity statistics along the solid particle trajectories is put into evidence, and its relevance in the derivation of Lagrangian transport models is discussed.
\end{abstract}

PACS numbers: 47.27.Qb 47.55.Kf 02.50.Ey 


\section{Introduction}

A heavy particle in a turbulent flow moves along a trajectory, which does not coincide with that of the fluid parcel where it lied initially [1]. This phenomenon is controlled by the particle trajectory relaxation time $\tau_{S}$, which, in general, is a function of the difference between the velocities $\mathbf{v}$ and $\mathbf{u}$ respectively of the particle and of the fluid at the particle position. To lowest order in the particle Reynolds number $R e_{p}, \tau_{S}$ is given by the Stokes time. (For a spherical particle, the particle Reynolds number and the Stokes time are defined respectively as $R e_{p}=|\mathbf{u}-\mathbf{v}| a / \nu$ and $\tau_{S}=\frac{2 a^{2}}{9 \nu}\left|1-\rho / \rho_{0}\right|$ with $\rho$ and $\rho_{0}$ the particle and fluid density, $\nu$ the fluid kinematic viscosity and $a$ the particle diameter). Divergence of fluid and particle trajectories receive contribution both from external forces (e.g. gravity) acting on the particle and acceleration along a fluid trajectory; $\mathbf{v}$ obeys therefore, to lowest order in $R e_{p}$ and $\rho_{0} / \rho$, the linear equation:

$$
\dot{\mathbf{v}}=\tau_{S}^{-1}(\mathbf{u}-\mathbf{v})+\mathbf{g}
$$

with $\left(\rho-\rho_{0}\right) \mathbf{g}$ the force acting on the particle. Accelerations produced by non-uniformity or nonstationarity of the mean flow may be reabsorbed into the term $\mathbf{g}$, after redefinition of $\mathbf{u}$ and $\mathbf{v}$, through a Galilean shift to the reference frame of the mean flow.

Starting from the work of Csanady [2], much of the interest has been in the role of gravity, with the conclusion that its main effect on turbulent diffusion is a renormalization of the correlation time. A particle, in the presence of an external force, will drift with respect to the fluid with an average velocity $\tau_{S} \mathbf{g}$, a phenomenon known under the name of trajectory crossing, and this will cause a shorter time of permanence of a particle in a correlated region and a Lagrangian correlation time shorter than that of a fluid parcel.

This renormalization has been used for the derivation of Lagrangian models for heavy particles dispersion [3], but it is not clear whether a Markovian hypothesis for the velocity is indeed applicable to the case of a heavy particle. This problem, associated with the small turbulent scales being seen as frozen by the drifting particle, was not present in [4 5 , who dealt essentially with a turbulent flow with only large scales present. The same turbulent regime was considered in [6], based on an expansion around the case of an infinite inertia particle. In the Eulerian approach of [7], the same problem was avoided working in the limit of Stokes time shorter than the Kolmogorov time, which is the same assumption on which the classical theory by Tchen is based [8].

A different approach is to model fluid and solid particle trajectories on the same ground. The result of this line of reasoning is a class of Lagrangian models, of which, one of the prototypes is 
described in [9, 10]. The basic idea is to couple Eqn. (1) (or its more complex counterpart valid for arbitrary $R e_{p}$ ) with the equations for the solid particle trajectory and with the model equation for the trajectory of the fluid parcel where the particle lied initially. In high Reynolds numbers, one resorts typically to models which are Markovian in the Lagrangian velocity of the fluid parcel $\mathbf{u}^{\mathrm{F}}(t)$ (see e.g. [11). In this regime, the model equations would read, in the absence of a mean flow:

$$
\left\{\begin{array}{l}
\dot{\mathbf{x}}=\mathbf{v} ; \quad \dot{\mathbf{x}}^{\mathrm{F}}=\mathbf{u}^{\mathrm{F}} \\
\dot{\mathbf{u}}^{\mathrm{F}}=\mathbf{a}\left(\mathbf{u}^{\mathrm{F}}, \mathbf{x}^{\mathrm{F}}\right)+\left(C_{0} \bar{\epsilon}\left(\mathbf{x}^{\mathrm{F}}\right)\right)^{\frac{1}{2}} \boldsymbol{\xi} ; \quad\left\langle\xi_{i}(t) \xi_{j}(0)\right\rangle=\delta_{i j} \delta(t)
\end{array}\right.
$$

where $a\left(\mathbf{u}^{\mathrm{F}}, \mathbf{x}^{\mathrm{F}}\right)$ is of the order of $u^{\mathrm{F}} / \tau_{L}\left(\mathbf{x}^{\mathrm{F}}\right)$, with $\tau_{L}\left(\mathbf{x}^{\mathrm{F}}\right)$ the turbulent integral time, $\bar{\epsilon}\left(\mathbf{x}^{\mathrm{F}}\right)$ is the mean turbulent dissipation and $C_{0}$ is an empirical constant, with values lying between 2 and 7 depending on the kind of flow [12]; $\mathbf{x}^{\mathrm{F}}$ is the coordinate of the fluid parcel for which initially: $\mathbf{x}^{\mathrm{F}}=\mathbf{x}$. After a time $\Delta$, whose determination is part of the modelling effort, a new fluid parcel is considered, thus setting again $\mathbf{x}^{\mathrm{F}}=\mathbf{x}$ and the process is repeated; in the meanwhile, when $\mathbf{x}^{\mathrm{F}} \neq \mathbf{x}$, the problem is to calculate from $\mathbf{u}^{\mathrm{F}}$ the fluid velocity $\mathbf{u}$ at the solid particle position $\mathbf{x}$.

This approach is able in principle to deal with both regimes of strong external forcing and dominant trajectory crossing, and of random trajectory separation produced solely by finite inertia and particles being unable to follow the rapid turbulent fluctuations. Obtaining the fluid velocity at the particle position turns out to be the difficult part of the problem. In [9, 10, the difference $\mathbf{u}-\mathbf{u}^{\mathrm{F}}$ was calculated only at the discrete times $t_{n}=n \Delta$, with the values of $\mathbf{u}-\mathbf{u}^{\mathrm{F}}$ at different $t_{n}$ assumed uncorrelated. However, although there have been other attempts in the direction of Markov modelling [13], the statistics for $\mathbf{u}$ appears to be non-Markovian, in general, both in time and in the separation $\mathbf{x}-\mathbf{x}^{\mathrm{F}}$. Only recently [14, 15], has the importance of anomalous scaling for the velocity along solid particle trajectories been recognized. However, the mechanism for its production was only partially discussed and no prediction for the dependence of the scaling exponent on the flow parameters was provided.

The problems associated with the non-Markovian nature of the fluid velocity $\mathbf{u}$ are further complicated in the case of weak external forces, with turbulent fluctuations and inertia dominating the process of trajectory separation. Analysis of this problem and of its consequence in terms of constraints on the possible form of turbulent transport models will be the subject of this paper.

\section{Scaling analysis}

One can see a turbulent flow as a superposition of vortices of different size $r$, which, at high 
Reynolds numbers, obey Kolmogorov scaling. In terms of the space (Eulerian) longitudinal structure function, this means, for $r \ll L$, with $L$ of the order of the size of the largest eddies:

$$
u_{r}^{2}=\left\langle\left[u_{r}^{\mathrm{E}}(\mathbf{x}+\mathbf{r}, t)-u_{r}^{\mathrm{E}}(\mathbf{x}, t)\right]^{2}\right\rangle=C_{K}(\bar{\epsilon} r)^{\frac{2}{3}}=u_{L}^{2}\left(\frac{r}{L}\right)^{\frac{2}{3}}
$$

with $\mathbf{u}^{\mathrm{E}}(\mathbf{x}, t)$ the Eulerian velocity field, $C_{K} \simeq 2$ the Kolmogorov constant and $u_{L}^{2}=\left\langle\left[u_{i}^{\mathrm{E}}\right]^{2}\right\rangle$. In terms of the time (Lagrangian) structure function, for $t \ll \tau_{L}$ :

$$
\left\langle\left[u_{i}^{\mathrm{F}}(t)-u_{i}^{\mathrm{F}}(0)\right]^{2}\right\rangle=C_{0} \bar{\epsilon} t=u_{L}^{2} \frac{t}{\tau_{L}}
$$

with $\tau_{L}$ of the order of the Lagrangian correlation time $T_{L}$ :

$$
T_{L}=u_{L}^{-2} \int_{0}^{\infty} \mathrm{d} t\left\langle u_{i}^{\mathrm{F}}(t) u_{i}^{\mathrm{F}}(0)\right\rangle
$$

The quantities $L, \tau_{L}$ and $u_{L}$ identify integral length, time and velocity scales of the flow, and, from Eqns. (3-4), an eddy turnover time can be defined in the standard way:

$$
\tau_{r}=\tau_{L}\left(\frac{r}{L}\right)^{\frac{2}{3}}
$$

Consider a solid particle, which at time $t=0$ is in $\mathbf{x}_{0}$ moving with speed $\mathbf{v}_{0}$ and to fix the ideas imagine first that no external forces are present. The trajectory separation will be given by:

$$
\Delta \mathbf{x}(t)=\mathbf{x}(t)-\mathbf{x}^{\mathrm{F}}(t)=\int_{0}^{t} \mathrm{~d} t^{\prime}\left[\mathbf{v}\left(t^{\prime}\right)-\mathbf{u}^{\mathrm{F}}\left(t^{\prime} \mid \mathbf{x}_{0}, 0\right)\right]
$$

where $\mathbf{u}^{\mathrm{F}}\left(t \mid \mathbf{x}_{0}, 0\right)$ is the fluid velocity of the fluid parcel, which at time $t=0$ was in $\mathbf{x}_{0}$.

Asymptotically, for $\tau_{S} / \tau_{L}, \tau_{\eta} / \tau_{S} \rightarrow 0$, where $\eta=\nu^{\frac{3}{4}} \bar{\epsilon}^{\frac{1}{4}}$ is the Kolmogorov length and $\tau_{\eta}$ is the associated Kolmogorov time, we can distinguish three phases in the separation process. The central role is played by the velocity and length scales of the vortices with life-time $\tau_{S}$, namely, for $\tau_{L}>\tau_{S}$

$$
u_{S}=u_{L}\left(\tau_{S} / \tau_{L}\right)^{\frac{1}{2}}, \quad S=L\left(\tau_{S} / \tau_{L}\right)^{\frac{3}{2}}=u_{S} \tau_{S}
$$

$\tau_{S}$ and $u_{S}$ will appear to be the time and velocity scale of the velocity difference $\mathbf{u}-\mathbf{v}$.

If $\mathbf{u}_{0} \equiv \mathbf{u}(0)=\mathbf{u}^{\mathrm{F}}\left(0 \mid \mathbf{x}_{0}, 0\right)$ is the initial fluid velocity and $\left|\mathbf{v}_{0}-\mathbf{u}_{0}\right| \ll u_{S}$, there will be an initial phase, in which the particle sees the turbulent field as frozen and the separation process is ballistic:

$$
\Delta \mathbf{x}(t) \simeq\left(\mathbf{v}_{0}-\mathbf{u}_{0}\right) t
$$

In fact, if $\Delta x(t)$ is so small that vortices of size $\Delta x(t)$ have characteristic velocity $u_{\Delta x(t)} \ll\left|\mathbf{v}_{0}-\mathbf{u}_{0}\right|$, it will also be $\frac{\Delta x(t)}{\left|\mathbf{u}^{\mathrm{F}}-\mathbf{v}\right|} \ll \tau_{\Delta x(t)}$, i.e. the crossing time of the vortex by the solid particle will be 
much shorter than the eddy turnover time of that vortex. The contribution from this vortex to $\Delta x$ will thus be $u_{\Delta x(t)} \frac{\Delta x(t)}{\left|\mathbf{u}^{\mathrm{F}}-\mathbf{v}\right|} \ll \Delta x(t)$ and then $\Delta x(t) \simeq\left|\mathbf{u}_{0}-\mathbf{v}_{0}\right| t$. This phase will last until $u_{\Delta x(t)} \sim\left|\mathbf{u}_{0}-\mathbf{v}_{0}\right|$, when the contribution from the vortices begins to be important. For the Kolmogorov spectrum described by Eqn. (2), this leads to a cross-over time:

$$
\tau_{B} \sim C_{K}^{-\frac{3}{2}} \bar{\epsilon}^{-1}\left|\mathbf{v}_{0}-\mathbf{u}_{0}\right|^{2}
$$

and for $\left|\mathbf{v}_{0}-\mathbf{u}_{0}\right| \ll u_{S}$, it will also be $\tau_{B} \ll \tau_{S}$.

In the next phase, for $\tau_{B} \ll t \ll \tau_{S}$, the separation process will receive two contributions: one from the velocity difference across a vortex of size $\Delta x(t)$, the other from the fact that the solid particle is unable to follow the fluid on time scales much shorter that $\tau_{S}$. From the relaxation equation nature of Eqn. (1), these contributions are both of the same order, and the end result is that the particles will separate with a relative velocity of the order of $u_{\Delta x(t)}$. This leads to a process of Richardson diffusion $\left\langle\Delta x^{2}(t)\right\rangle \propto t^{3}$, although with a coefficient different from that occurring in the case of a pair of fluid particles. Now, for most choices of initial conditions: $\left|\mathbf{v}_{0}-\mathbf{u}_{0}\right| \sim u_{S}$, the ballistic phase will end at $t \sim \tau_{B} \sim \tau_{S}$ and the Richardson diffusion phase will be absent. The same will occur when $\left|\mathbf{v}_{0}-\mathbf{u}_{0}\right|>u_{S}$; the ballistic phase will end when the solid particle trajectory relaxes, which happens, again, when $\tau_{B} \sim \tau_{S}$.

In the end phase, for $t \gg \tau_{S}$, the solid particle will deviate from the fluid parcel it crosses at the time $t$, with a rate much smaller than the rate of separation from the fluid parcel it crossed at time zero. In fact, from Eqn. (1), the difference $\mathbf{v}-\mathbf{u}$ will saturate at $t \sim \tau_{S}$, when its magnitude is $O\left(u_{S}\right)$; for $t>\tau_{S}, \mathbf{v}-\mathbf{u}$ will continue to fluctuate with amplitude $u_{S}$ and correlation time $\tau_{S}$. In this end phase, the equation for $\Delta \mathbf{x}$ will have the form:

$$
\Delta \dot{\mathbf{x}}(t) \simeq \mathbf{u}^{\mathrm{F}}\left(t \mid \mathbf{x}\left(\tau_{S}\right), \tau_{S}\right)-\mathbf{u}^{\mathrm{F}}\left(t \mid \mathbf{x}^{\mathrm{F}}\left(\tau_{S}\right), \tau_{S}\right)
$$

i.e. Richardson law for the separation of two fluid particles, which, at time $\tau_{S}$, were distant $\Delta x\left(\tau_{S}\right)=\left|\mathbf{x}\left(\tau_{S}\right)-\mathbf{x}^{\mathrm{F}}\left(\tau_{S}\right)\right| \sim S$. Of course, when $\Delta x(t)>L$ (and $t>\tau_{L}$ ), the fluctuations at $\mathbf{x}$ and $\mathbf{x}_{F}$ will decorrelate and Richardson diffusion will turn into normal diffusion. In the extreme case of very fast turbulent fluctuations, i.e. $\tau_{L}<\tau_{S}$, the Richardson diffusion phase may be absent and separation will cross over directly to a regime of normal diffusion, without passing through the regime described by Eqn. (11), with $|\mathbf{u}-\mathbf{v}|$ saturating to $u_{L}$ instead of to $u_{S}$.

The picture is complicated a bit by the presence of external forcing on the particle; this leads 
to new characteristic scales for velocity, length and time:

$$
u_{G}=g \tau_{S}, \quad G=L\left(g \tau_{S} / u_{L}\right)^{3} \quad \text { and } \quad \tau_{G}=\tau_{L}\left(g \tau_{S} / u_{L}\right)^{\frac{3}{2}}
$$

associated respectively with the drift of the particle in the fluid, and with the size and lifetime of the eddies having this characteristic velocity. The relative importance of external forces and of random acceleration by turbulence is fixed by the ratio $u_{G} / u_{S}$. Both limit regimes $\tau_{S}=0, u_{G} \neq 0$ and $\tau_{S}>0, g=0$ are physically realizable, an example of the first one being that of charged buoyant particles $\left(\rho=\rho_{0}\right)$ in a strong electric field. External forcing will have a negligible effect if $u_{G}<\min \left(u_{S}, u_{L}\right)$, i.e., from Eqn. (12), if:

$$
u_{L}>\frac{g \tau_{S}}{\min \left(1, \tau_{S} / \tau_{L}\right)^{\frac{1}{2}}}
$$

Thus, inertia may be dominant, when turbulent fluctuations are so strong and fast that $u_{L}>g \tau_{S}$ and $\tau_{L}<\tau_{S}$ (for $\rho / \rho_{0} \gg 1$, in which case external forces are an important factor, this happens typically in the vicinity of obstacles) or otherwise, when the particles are small and the Stokes time is short enough: $u_{L}>g\left(\tau_{S} \tau_{L}\right)^{\frac{1}{2}}$.

In the presence of external forces, the difference $\mathbf{u}-\mathbf{v}$ will fluctuate with amplitude $u_{S}$ and time scale $\tau_{S}$, around $\mathbf{u}_{G}$ instead of around zero. For $u_{G} \ll u_{S}$, trajectory separation develops in the same sequence of phases as in the case without forces; the time $\tau_{G}<\tau_{S}$ identifies only the value of the crossover time $\tau_{B}$ corresponding to $\left|\mathbf{u}_{0}-\mathbf{v}_{0}\right| \sim u_{G}$.

When external forcing dominates over the effect of inertia, i.e. when $u_{G} \gg u_{S}$, the sequence of phases is altered. The initial phase $t<\tau_{B}$ remains ballistic in nature. Also the next one, for $\tau_{B} \ll t \ll \tau_{S}$, is not modified; since $g t \ll u_{\Delta x(t)}$, the external force contribution is small and the particle separation follows the same pattern a la Richardson as in the $g=0$ case. For $\tau_{S} \ll t \ll \tau_{G}$, however, a new phase will occur: the particle is drifting with velocity $u_{G}$ and the contribution to separation from vortices of size $\Delta x(t)$ is negligible: $\Delta x(t) \ll G$ and $u_{\Delta x(t)} \ll u_{G}$. The end phase of pure Richardson diffusion described by Eqn. (11) starts only when $t>\tau_{G}$, so that $u_{\Delta x(t)}>u_{G}$, but may be absent in the case of external forces dominating over turbulence: $u_{G}>u_{L}$. In this case, also the end phase will be dominated by the drift, with superimposed a normal diffusion correction produced by eddies at scales $L$. While in the absence of gravity, the cross-over to fluid-like behaviors occurred at the space scale $S$, in the present case the transition occurs at the scale $G$, which, since $u_{G} \gg u_{S}$, is larger than $S$.

In the three cases of dominant inertia, intermediate (in which external forces dominate inertia, 
but are dominated by turbulence), and external forces dominating over both inertia and turbulence, the separation process will develop in the following possible sequences of phases:

Dominant inertia $\tau_{G}<\tau_{S}$ : Ballistic $\left(t<\tau_{B}\right) \rightarrow$ Modified Richardson $\left(\tau_{B}<t<\tau_{S}\right) \rightarrow$ Richardson $\left(\tau_{S}<t<\tau_{L}\right) \rightarrow$ Normal $\left(t>\tau_{L}\right)$;

Intermediate $\tau_{S}<\tau_{G}<\tau_{L}:$ Ballistic $\left(t<\tau_{B}\right) \rightarrow$ Modified Richardson $\left(\tau_{B}<t<\tau_{S}\right) \rightarrow$ Drift $\left(\tau_{S}<t<\tau_{G}\right) \rightarrow$ Richardson $\left(\tau_{G}<t<\tau_{L}\right) \rightarrow$ Normal $\left(t>\tau_{L}\right)$.

Dominant External Forces $\tau_{G}>\tau_{L}$ : Ballistic $\left(t<\tau_{B}\right) \rightarrow$ Modified Richardson $\left(\tau_{B}<t<\right.$ $\left.\tau_{S}\right) \rightarrow \operatorname{Drift}\left(t>\tau_{S}\right)$

The considerations that have been carried on so far correspond to the intuitive fact that the turbulent small scales, i.e. those for which $l<\max (S, G)$, are unable to transport the solid particles. For $l<S$ this occurs because of inertia; for $S<l<G$, it is the external forcing produced trajectory crossing which is responsible for this. In the case of the large scales $l>\max (S, G)$, the contrary occurs: one has $|\mathbf{u}-\mathbf{v}| \sim \max \left(u_{S}, u_{G}\right)<u_{l}$ and the deviation of the particle trajectory from that of a fluid parcel on the scale of a vortex of size $l$ will be $\sim u_{S, G}\left(\tau_{S, G} \tau_{l}\right)^{\frac{1}{2}}<l$ and the solid particle will behave like a fluid one.

This scale separation of the turbulent fluctuations and the the subdivision in phases of the trajectory separation process, has a counterpart in the self-diffusion $\left\langle\left|\mathbf{u}\left(t \mid \mathbf{x}_{0}, 0\right)-\mathbf{u}_{0}\right|^{2}\right\rangle$, which is the relevant quantity to determine the transport properties of the particles $\left[\mathbf{u}\left(t \mid \mathbf{x}_{0}, 0\right)\right.$ is the fluid velocity sampled by the solid particle, which at time $t=0$ was in $\mathbf{x}_{0}$ ]. Following [10] we can split the velocity difference $\mathbf{u}\left(t \mid \mathbf{x}_{0}, 0\right)-\mathbf{u}_{0}$ into the different contributions along, and transversal to fluid trajectories. The first contribution is just the Lagrangian change $\mathbf{u}^{\mathrm{F}}\left(t \mid \mathbf{x}_{0}, 0\right)-\mathbf{u}_{0}$; the second one $\mathbf{u}\left(t \mid \mathbf{x}_{0}, 0\right)-\mathbf{u}^{\mathrm{F}}\left(t \mid \mathbf{x}_{0}, 0\right)=\mathbf{u}^{\mathrm{E}}(\mathbf{x}(t), t)-\mathbf{u}^{\mathrm{E}}\left(\mathbf{x}^{\mathrm{F}}(t), t\right)$ is a complex quantity feeling both effects of trajectory separation and of the relative diffusion of fluid elements. In the ballistic regime, using Eqn. (3), we have:

$$
\begin{aligned}
& \mathbf{u}\left(t \mid \mathbf{x}_{0}, 0\right)-\mathbf{u}^{\mathrm{F}}\left(t \mid \mathbf{x}_{0}, 0\right) \simeq \mathbf{u}^{\mathrm{E}}\left(\mathbf{x}_{0}+\mathbf{v}_{0} t, t\right)-\mathbf{u}^{\mathrm{E}}\left(\mathbf{x}_{0}+\mathbf{u}_{0} t, t\right) \\
& \sim u_{\left|\mathbf{u}_{0}-\mathbf{v}_{0}\right| t} \sim u_{L}\left(\left|\mathbf{u}_{0}-\mathbf{v}_{0}\right| t / L\right)^{\frac{1}{3}}
\end{aligned}
$$

while, from Eqn. (4):

$$
\mathbf{u}^{\mathrm{F}}\left(t \mid \mathbf{x}_{0}, 0\right)-\mathbf{u}_{0} \sim \frac{u_{L} t}{\tau_{L}} \ll u_{L}\left(\frac{\left|\mathbf{u}_{0}-\mathbf{v}_{0}\right| t}{L}\right)^{\frac{1}{3}}
$$

Therefore:

$$
\left\langle\left|\mathbf{u}\left(t \mid \mathbf{x}_{0}, 0\right)-\mathbf{u}_{0}\right|^{2}\right\rangle \sim C_{K}\left(\bar{\epsilon}\left|\mathbf{v}_{0}-\mathbf{u}_{0}\right| t\right)^{\frac{2}{3}} \sim C_{K}\left(\bar{\epsilon} u_{A} t\right)^{\frac{2}{3}}
$$


where $t \ll \tau_{B}$ and $A=S, G$.

In the opposite regime $t \gg \tau_{S, G}$, from Eqn. (11), the transverse separation will be dominated by the relative diffusion of fluid elements: $\mathbf{u}\left(t \mid \mathbf{x}_{0}, 0\right)-\mathbf{u}^{\mathrm{F}}\left(t \mid \mathbf{x}_{0}, 0\right) \simeq \mathbf{u}^{\mathrm{F}}\left(t \mid \mathbf{x}\left(\tau_{S}\right), \tau_{S}\right),-\mathbf{u}^{\mathrm{F}}\left(t \mid \mathbf{x}^{\mathrm{F}}\left(\tau_{S}\right), \tau_{S}\right)$ while the "longitudinal" component remains $\mathbf{u}^{\mathrm{F}}\left(t \mid \mathbf{x}_{0}, 0\right)-\mathbf{u}_{0} \sim\left(C_{0} \bar{\epsilon} t\right)^{\frac{1}{2}}$. Also the first component obeys normal scaling, but its presence comes simply from having taken a wrong initial condition for $\mathbf{u}\left(t \mid \mathbf{x}_{0}, 0\right) \simeq \mathbf{u}^{\mathrm{F}}\left(t \mid \mathbf{x}\left(\tau_{S}\right), \tau_{S}\right)$. Said in a different way, the transverse component is not independent of the longitudinal one, and they sum together in such a way that the total amplitude is equal to that of the longitudinal piece. The total velocity variation is therefore:

$$
\left\langle\left|\mathbf{u}\left(t \mid \mathbf{x}_{0}, 0\right)-\mathbf{u}_{0}\right|^{2}\right\rangle \sim C_{0} \bar{\epsilon} t \quad \tau_{L} \gg t \gg \tau_{S}, \tau_{G}
$$

i.e., for long times and turbulence strong enough to prevent drift dominance at large $t$, the selfdiffusion properties of fluid and solid particles will be the same.

In the middle range $\tau_{B} \ll t \ll \tau_{S}$, all the contributions to $\mathbf{u}\left(t \mid \mathbf{x}_{0}, 0\right)-\mathbf{u}_{0}$ are of the same order; these are the Lagrangian change $\mathbf{u}^{\mathrm{F}}\left(t \mid \mathbf{x}_{0}, 0\right)-\mathbf{u}_{0}$, the one from Richardson diffusion starting at times $\tau_{B} \ll t: \mathbf{u}^{\mathrm{F}}\left(t \mid \mathbf{x}\left(\tau_{B}\right), \tau_{B}\right)-\mathbf{u}^{\mathrm{F}}\left(t \mid \mathbf{x}^{\mathrm{F}}\left(\tau_{B}\right), \tau_{B}\right)$, and the remnant $\mathbf{u}\left(t \mid \mathbf{x}\left(\tau_{B}\right), \tau_{B}\right)-\mathbf{u}^{\mathrm{F}}\left(t \mid x\left(\tau_{B}\right), \tau_{B}\right)$, which is due to trajectory separation. In fact, $t$ identifies a scale $l$, through the relation $\tau_{l}=t$, which is the size of the vortices contributing most both to $\mathbf{u}\left(t \mid \mathbf{x}_{0}, 0\right)-\mathbf{u}_{0}$, to Richardson diffusion and to the trajectory separation $|\mathbf{u}(t)-\mathbf{v}(t)| \sim u_{l}$. Even after elimination of the Richardson diffusion by choosing a different Lagrangian path, the difference $\mathbf{u}\left(t \mid \mathbf{x}_{0}, 0\right)-\mathbf{u}_{0}$ will receive a contribution proportional to $u_{l}$ due to the $O(l)$ displacement between the solid and fluid particle trajectory. This is of the same order as the Lagrangian piece $\mathbf{u}^{\mathrm{F}}\left(t \mid \mathbf{x}_{0}, 0\right)-\mathbf{u}_{0}$, associated with the time evolution of a size $l$ vortex, which is $O\left(u_{l}\right)$ in the time $t \sim \tau_{l}$. The end result will be again, the normal behavior for velocity self-diffusion, described in Eqn. (15), but with a renormalized $C_{0}$ to account for the trajectory separation $|\mathbf{u}(t)-\mathbf{v}(t)| \sim u_{l}$.

\section{Transport by a synthetic turbulent field}

One of the difficulties in studying the divergence of solid and fluid particles is that the process is affected simultaneously by Eulerian and Lagrangian properties of the turbulent flow. To simulate numerically the trajectory of a single solid particle, one would expect therefore that a whole advecting field should be generated. Clearly, this is as difficult as generating a high Reynolds number turbulent flow by means of a DNS (direct numerical simulation) of the Navier-Stokes equation. The strategy to bypass this problem, while working in high Reynolds number conditions, 
has been to generate numerically an advecting flow only in the neighborhood of the solid particle. This flow satisfies a restricted set of properties of real turbulence, namely, incompressibility, Kolmogorov scaling for the space structure function and normal scaling for the Lagrangian time structure function, as described by Eqn. (4).

The flow was generated by means of a superposition of vortices with the following properties (more details are given in the Appendix):

i The vortices are distributed in a sequence of shells: those in shell $n$ have size $l \in$ $\left[2^{-n-1}, 2^{-n}\right]$ and are distributed uniformly in the logarithm of scale [16]. In this way, if $N+1$ is the total number of shells: $0 \leq n \leq N$, and thus a Kolmogorov length $\eta=2^{-N-1}$ and an effective Reynolds number $R e=L / \eta \sim 2^{N+1}$ are introduced in a natural way.

ii The vortices have a finite lifetime $\bar{\tau}_{l}=\beta l^{\frac{2}{3}}$, with $\beta$ a fixed constant, and are generated in the neighborhood of the particle in such a way that the particle lies on the average in the support of only one vortex per shell. The radius of this neighborhood scales linearly with the shell scale, so that all shells have, on the average, the same number of vortices.

iii A vortex in shell $n$ translates rigidly with speed equal to the value at its center of the velocity field produced by all vortices in shells $n^{\prime}<n$.

For the kind of analysis of scaling and crossover behaviors we are interested in, two dimensions are expected to be sufficient. In addition, working in 2D allows to neglect difficulties associated with the choice of eddy shape, which here is taken as circular.

The idea of mimicking a turbulent flow with a superposition of rigid vortices, whose dynamics imitate as much as possible that of the real ones is not new [17] (see [18] for an application including the effect of Lagrangian sweep). In our case, thanks to the limited number of vortices per shell, this allows a drastic reduction in the number of degrees of freedom in the system. One more advantage is associated with the fact that a flow space structure is present and incompressibility can be enforced. This prevents the difficulties present in more standard Lagrangian stochastic models, which are associated with the need of a well mixed condition [1] imposed from the outside. The same problem occurs in the random walk model considered in [19, 20], in which the vortices are modelled as scattering centers for the particles. 
The same approach based on the use of a superposition of vortices localized close to a single particle can be utilized to analyze the separation of particle pairs. The separation of trajectories in the two cases of a pair of fluid particles $\left(\tau_{S}=0\right)$ and of a $\tau_{S} / \tau_{L}=0.26$ solid particle and a fluid one are shown in Fig. 1 below.

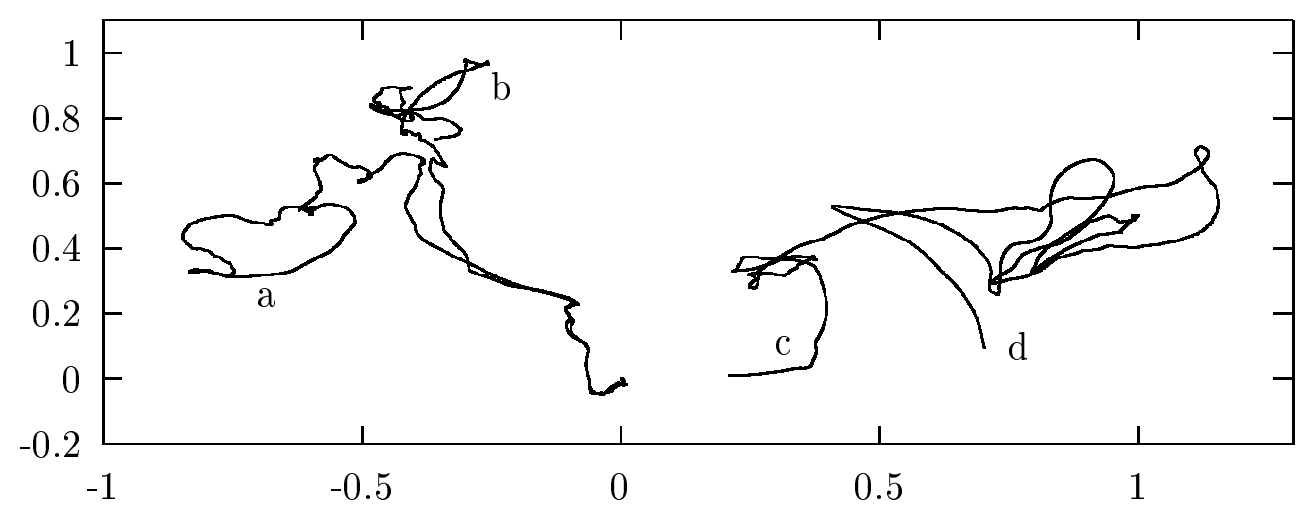

Figure 1: Two pairs of trajectories separating in a turbulent flow, in the absence of external forces. Effective Reynolds number $L / \eta \simeq 10^{5}$ (17 shells). Trajectories $a, b$ and $c$ refer to fluid particles; $d$ is the trajectory of a solid particle with $\tau_{S} / \tau_{L}=0.26$. Notice the smoother path followed in $d$. Notice also the long transient in the first pair, where both particle are fluid and all the separation is due to fluid trajectory divergence.

In the first case, an initial separation $0.25 \eta$ was adopted; notice, however, the long transient associated with the particles lying at "viscous range" separations. The corresponding relative diffusion in velocity: $\left\langle\left|\Delta u_{i}(t)\right|^{2}\right\rangle=\left\langle\left[u_{i}\left(t \mid \mathbf{x}_{0}, 0\right)-u_{i}^{\mathrm{F}}\left(t \mid \mathbf{x}_{0}, 0\right)\right]^{2}\right\rangle$ and in space $\left\langle\left|\Delta x_{i}(t)\right|^{2}\right\rangle=$ $\left\langle\left[x_{i}\left(t \mid \mathbf{x}_{0}, 0\right)-x_{i}^{\mathrm{F}}\left(t \mid \mathbf{x}_{0}, 0\right)\right]^{2}\right\rangle$ are shown in Figs. 2 and 3.

The parameter $\beta$ has been chosen to maximize the ratio $C_{K}^{\frac{3}{2}} / C_{0}$, and therefore the velocity variation due to trajectory separation. This choice of parameters resulted in: $C_{K}^{-\frac{3}{2}} C_{0} \simeq 2.828$. In the artificial flow considered, the turbulent dissipation $\bar{\epsilon}$ is clearly undefined, and the constants $C_{K}$ and $C_{0}$ cannot be determined individually. Assuming $C_{K}=2$, as in three dimensional turbulence, would give $C_{0} \simeq 8$, which is rather large, as are small the values of the constants $c$ and $\tilde{c}$ entering the expressions for velocity and coordinate relative diffusion $\left\langle\left|\Delta u_{i}(t)\right|^{2}\right\rangle=c \bar{\epsilon} t$ and $\left\langle\left|\Delta x_{i}(t)\right|^{2}\right\rangle=\tilde{c} \bar{\epsilon} t^{3}$. One finds: $c / C_{0} \simeq 0.29$ and $\tilde{c} / C_{0}$, which would lead to: $c \simeq 2.3$ and $\tilde{c} \simeq 0.025$. All of these constants are characterized by a strong scatter of experimental values; if the accepted value for $C_{0}$ is around 4 [21], estimates for $\tilde{c}$ went as low as 0.05 [22], while recent measurements gave $\tilde{c} \simeq 0.25$ [23. The uncertainty in $c$ is magnified in $\tilde{c}$ due to the higher power of $t$, and a factor 10 in $\tilde{c}$ 


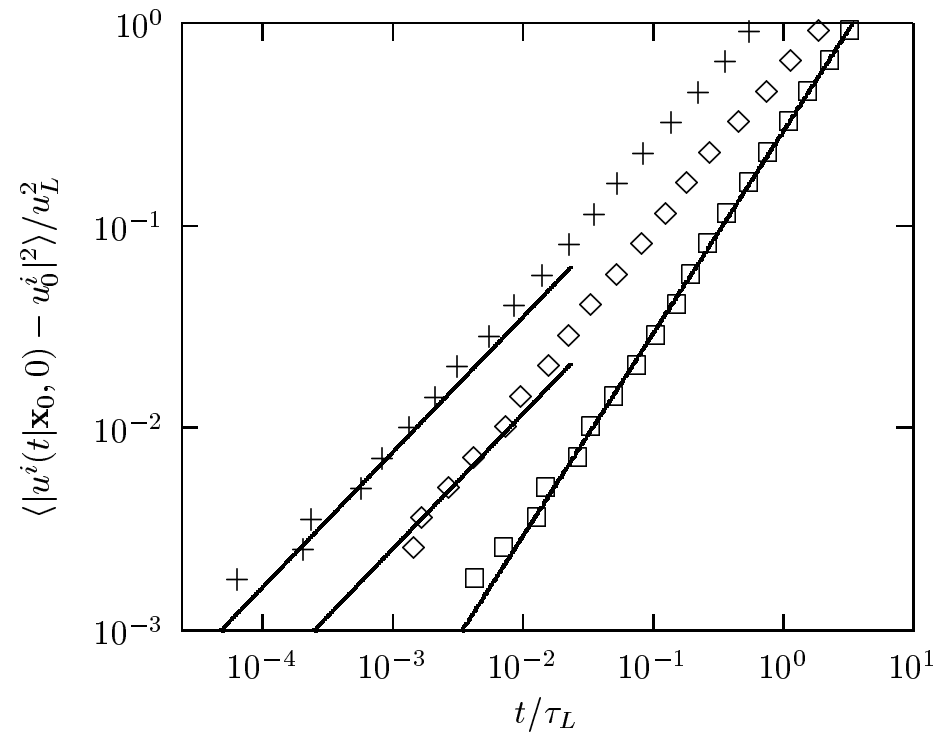

Figure 2: Relative diffusion of velocity: $\Delta \mathbf{u}(t)=\mathbf{u}\left(t \mid \mathbf{x}_{0}, 0\right)-\mathbf{u}^{\mathrm{F}}\left(t \mid \mathbf{x}_{0}, 0\right)$; effective Reynolds number $L / \eta \simeq 10^{5}$ (17 shells). $\square$ : fluid particle diffusion $\left(\tau_{S}=\tau_{G}=0\right)$, fitted with the linear law $\left\langle\left|\Delta u_{i}(t)\right|^{2}\right\rangle=0.29 \frac{u_{L}^{2} t}{\tau_{L}} . \diamond: \tau_{S}=0, \tau_{G} / \tau_{L}=0.01 ;+: \tau_{S} / \tau_{L}=0.13, \tau_{G}=0$. The lower end of the data are fitted with $t^{\frac{2}{3}}$ slopes.

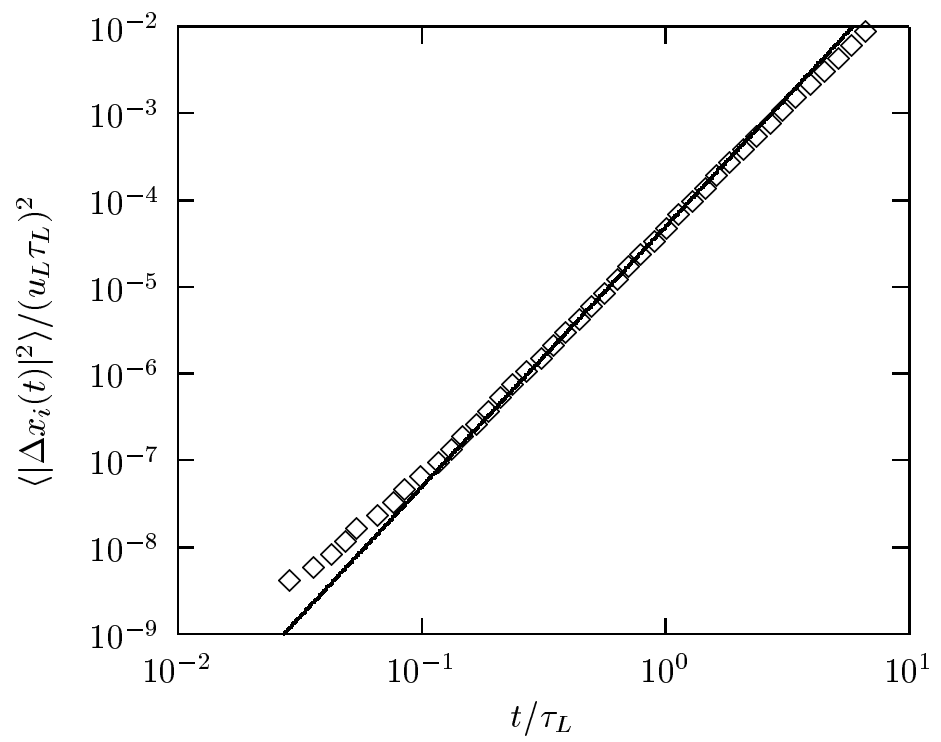

Figure 3: Richardson diffusion of a pair of fluid particles and fit by $\left\langle\left|\Delta x_{1}(t)\right|^{2}\right\rangle=0.003 \frac{u_{L}^{2} t^{3}}{\tau_{L}}$. Effective Reynolds number $L / \eta \simeq 10^{5}$ (17 shells). 
corresponds to a factor $\sim 2$ in $c$. Nonetheless, the small values of the ratios $c / C_{0}$ and $\tilde{c} / C_{0}$ (which, contrary to $c$ and $\tilde{c}$, are independent of $C_{K}$ ) indicate that, although the synthetic turbulence field satisfies the proper Lagrangian and Eulerian scaling, the ratio of relative diffusion to selfdiffusion may be smaller than in real flows. We must not forget, however, that the relevance to three dimensional turbulence of parameters such as e.g. $C_{K}^{\frac{3}{2}} / C_{0}$, obtained from a two dimensional synthetic field, may be somewhat limited.

The analysis of trajectory separation, contrary to that of the self-diffusion $\left\langle\left|\mathbf{u}\left(t \mid x_{0}, 0\right)-\mathbf{u}_{0}\right|^{2}\right\rangle$ is plagued by several difficulties. The most important one is the limitation in the sample size: small and large separations, in the relative diffusion case, are calculated from the same sample of trajectory pairs, while, in the self-diffusion case, the sample size is inversely proportional to the scale in exam. This limitation is particularly serious, since most of the information on the separation of solid particle trajectories is conditioned to the initial velocity difference. The second difficulty is the strong effect of transients occurring when the particles are very close and stick together, which is absent in the case of self-diffusion. In fact, as suggested in [24, to obtain the curves in Figs. 2 and 3, it was necessary to average over the logarithms of the doubling times of $\left|\mathbf{u}\left(t \mid \mathbf{x}_{0}, 0\right)-\mathbf{u}^{\mathrm{F}}\left(t \mid \mathbf{x}_{0}, 0\right)\right|$ and $\left|\mathbf{x}\left(t \mid \mathbf{x}_{0}, 0\right)-\mathbf{x}^{\mathrm{F}}\left(t \mid \mathbf{x}_{0}, 0\right)\right|$, instead of at fixed times, over $\mid \mathbf{u}\left(t \mid \mathbf{x}_{0}, 0\right)-$ $\mathbf{u}^{\mathrm{F}}\left(t \mid \mathbf{x}_{0}, 0\right) \mid$ and $\left|\mathbf{x}\left(t \mid \mathbf{x}_{0}, 0\right)-\mathbf{x}^{\mathrm{F}}\left(t \mid \mathbf{x}_{0}, 0\right)\right|$.

The self-diffusion properties of particles with different values of the ratio $\tau_{S} / \tau_{L}$ are shown in Figs. 4, and 5 for the two cases without and with external forces.

The time-scale of fluctuation for the difference $\mathbf{u}-\mathbf{v}$ appears to be of the order of $\tau_{G}$ and $\tau_{S}$, respectively, in the forced and unforced case. In the unforced case, the largest $\tau_{S} / \tau_{L}=2.6$ curve is basically indistinguishable from what would result from setting $\mathbf{v}=0$, i.e. $\tau_{S}=\infty$. (The short time behavior of this curve, incidently, gives then also an idea of the applicability of the Taylor frozen field hypothesis in the absence of a mean flow). In the forced case, only the largest value of the drift $u_{G}$ leads to an appreciable decrease in the correlation time for the fluid velocity sampled by the particle. In the other cases, in which $u_{L}>u_{G}$, the synthetic turbulence model is probably too crude to give any definite answer.

The inertial range is compressed passing from space to time (compare with Fig. 11 in the Appendix) and this has the consequence that crossover behaviors dominate also at very high Reynolds number. In order to observe cleaner scaling behaviors in the velocity self-diffusion, it would be necessary to condition the averages to the initial value of the difference $\mathbf{u}-\mathbf{v}$, as shown 


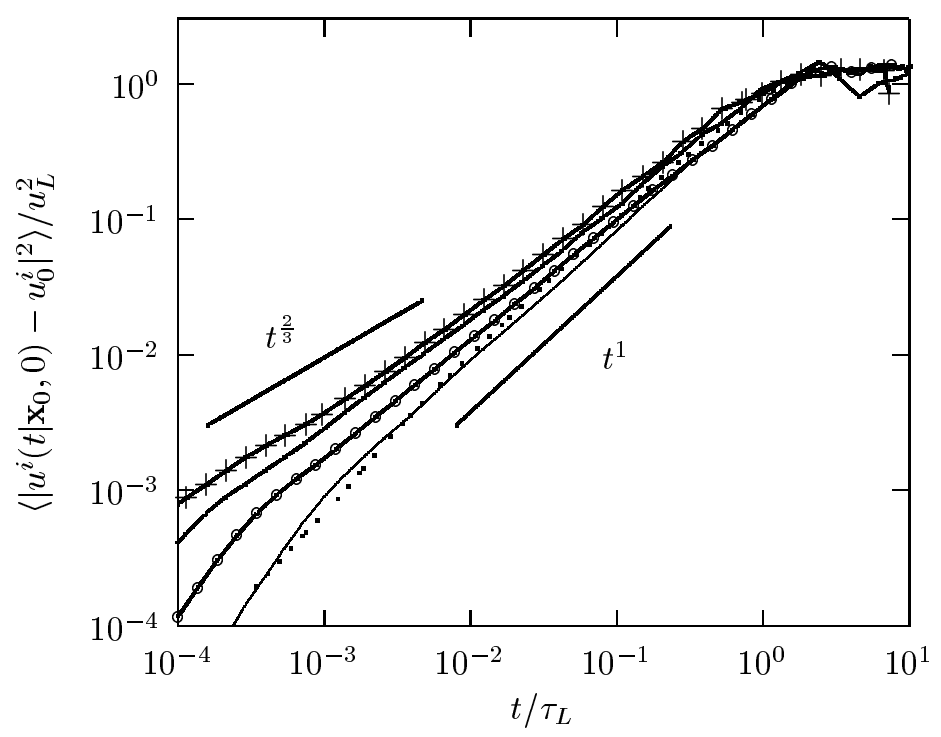

Figure 4: Velocity self-diffusion in the inertia dominated case $\left(u_{G}=0\right)$ for different values of $\tau_{S} / \tau_{L} \cdot{ }^{\prime}{ }^{-+-^{\prime}}: \tau_{S} / \tau_{L}=2.6$; heavy line: $\tau_{S} / \tau_{L}=0.26 ;^{\prime}-\circ-^{\prime}: \tau_{S} / \tau_{L}=0.026$; thin line: $\tau_{S} / \tau_{L}=$ 0.0026 ; dots: fluid particle. The Kolmogorov time-scale is $\tau_{\eta} / \tau_{L} \simeq 3.8 \times 10^{-4}$, corresponding to an effective Reynolds number $R e \simeq 2 \times 10^{5}$ (18 shells). In the various cases, we have, for $\sigma_{|\mathbf{u}-\mathbf{v}|}^{2} \equiv\left\langle|\mathbf{u}-\mathbf{v}|^{2}\right\rangle-u_{G}^{2}: \tau_{S} / \tau_{L}=0.0026: u_{L}^{-2} \sigma_{|\mathbf{u}-\mathbf{v}|}^{2}=0.0049 ; \tau_{S} / \tau_{L}=0.026: u_{L}^{-2} \sigma_{|\mathbf{u}-\mathbf{v}|}^{2}=0.047 ;$ $\tau_{S} / \tau_{L}=0.26: u_{L}^{-2} \sigma_{|\mathbf{u}-\mathbf{v}|}^{2}=0.39 ; \tau_{S} / \tau_{L}=2.6: u_{L}^{-2} \sigma_{|\mathbf{u}-\mathbf{v}|}^{2}=0.9$.

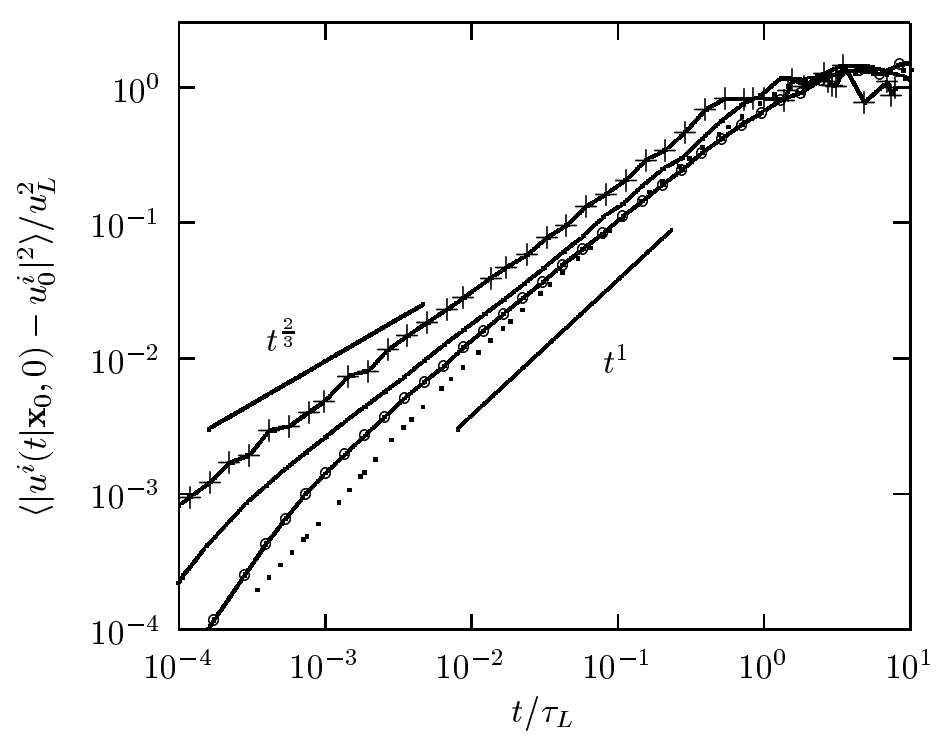

Figure 5: Velocity self-diffusion in the external forcing dominated regime $\left(\tau_{S}=0\right)$ for different values of $u_{G} / u_{L}$; same flow parameters as in Fig. $4 .{ }^{\prime}{ }_{-+-}{ }^{\prime}: u_{G} / u_{L}=1.83$; heavy line: $u_{G} / u_{L}=$ $0.09 ;{ }^{\prime}-\circ-{ }^{\prime}: u_{G} / u_{L}=0.01$; dots: unforced case. In the various cases we have: $u_{G} / u_{L}=1.83$ : $u_{L}^{-2} \sigma_{|\mathbf{u}-\mathbf{v}|}^{2}=1.7 ; u_{G} / u_{L}=0.09: u_{L}^{-2} \sigma_{|\mathbf{u}-\mathbf{v}|}^{2}=0.11 ; u_{G} / u_{L}=0.01: u_{L}^{-2} \sigma_{|\mathbf{u}-\mathbf{v}|}^{2}=0.015 ;$ 
in Fig. 6 in the unforced case.

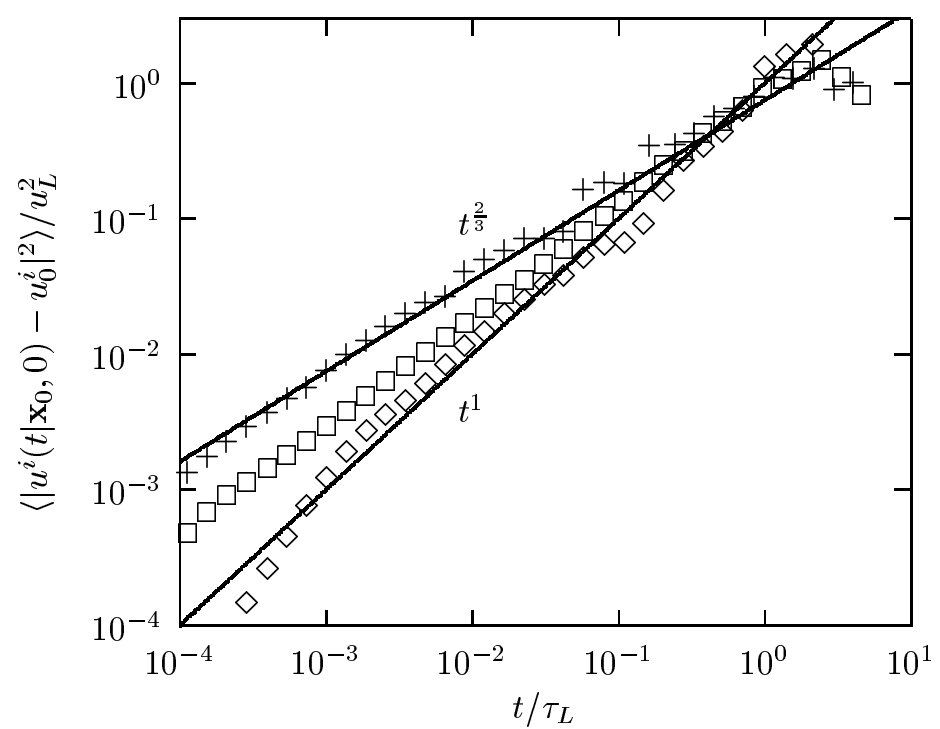

Figure 6: Velocity self-diffusion conditioned to different values of the ratio $\langle|\mathbf{u}-\mathbf{v}|\rangle^{-1}|\mathbf{u}-\mathbf{v}|$, for fixed $\tau_{S} / \tau_{L}=0.26, u_{G}=0$. Same flow characteristics as in Fig. $4 .^{\prime}+^{\prime}: 2<\langle|\mathbf{u}-\mathbf{v}|\rangle^{-1}|\mathbf{u}-\mathbf{v}|$. ${ }^{\prime} \square^{\prime}:\langle|\mathbf{u}-\mathbf{v}|\rangle^{-1}|\mathbf{u}-\mathbf{v}|<0.12 .^{\prime} \diamond^{\prime}$ : unconditioned. Notice how ballistic behaviors are enhanced in the two conditioned cases.

An example of cross-over behavior is the renormalization of the constant $C_{0}$ over the whole inertial range for values of $\tau_{S} / \tau_{L}$ as small as 0.0026 .

It is possible to have an idea of what is happening, at least in the unforced case, focusing on the separation of trajectories in a vortex of size $l>S$. The separation process is the result of the particle being accelerated by vortices of all the available scales $l^{\prime}$. From Eqn. (1), large vortices with $l^{\prime}>l$ will produce a constant velocity difference $u_{l^{\prime}} \tau_{S} / \tau_{l^{\prime}}$. The contribution to trajectory separation by a single vortex of size $l^{\prime}$, will be, in the lifetime $\tau_{l}$ :

$$
\Delta l\left(l^{\prime}\right) \sim \tau_{l} u_{l^{\prime}} \tau_{S} / \tau_{l^{\prime}} \sim l \tau_{S} / \tau_{l}\left(u_{l} / u_{l^{\prime}}\right)
$$

The contribution from vortices at scale $l^{\prime} \in[S, l]$, instead, is similar to a random walk, since each vortex $l^{\prime}$ produces an independent displacement of the particle in the time $\tau_{l}$; the total displacement produced in this time will be therefore:

$$
\Delta l\left(l^{\prime}\right) \sim l^{\prime} \tau_{S} / \tau_{l^{\prime}}\left(\tau_{l} / \tau_{l^{\prime}}\right)^{\frac{1}{2}} \sim l \tau_{S} / \tau_{l}
$$

Smaller vortices, of size $l^{\prime}<S$, produce altogether an $O(S)$ contribution to displacement. Exploiting the fact that the vortices are distributed uniformly in the logarithm of scale, and combining 
with Eqns. (16-17), the velocity shift will be:

$$
\sum_{S<l^{\prime}<l} \frac{\Delta l^{\prime} u_{l^{\prime}}}{l} \sim \sum_{S<l^{\prime}<l} \sum_{S<l^{\prime \prime}<l^{\prime}} \frac{\Delta l^{\prime}\left(l^{\prime \prime}\right) u_{l^{\prime}}}{l} \sim[\log (l / S)]^{2} \frac{u_{l} \tau_{S}}{\tau_{l}}
$$

The effect on the time velocity structure function will be, from Eqn. (6):

$$
\left\langle\left|\mathbf{u}\left(t \mid \mathbf{x}_{0}, 0\right)-\mathbf{u}_{0}\right|^{2}\right\rangle \sim C_{0} \bar{\epsilon} t\left(1+\frac{a \tau_{S}}{t}\left[\log \left(b t / \tau_{S}\right)\right]^{2}\right)
$$

with $a$ and $b$ constants. A fit of $\left\langle\left|\mathbf{u}\left(t \mid \mathbf{x}_{0}, 0\right)-\mathbf{u}_{0}\right|^{2}\right\rangle$ using Eqn. (19) is shown in Fig. 7. It is to

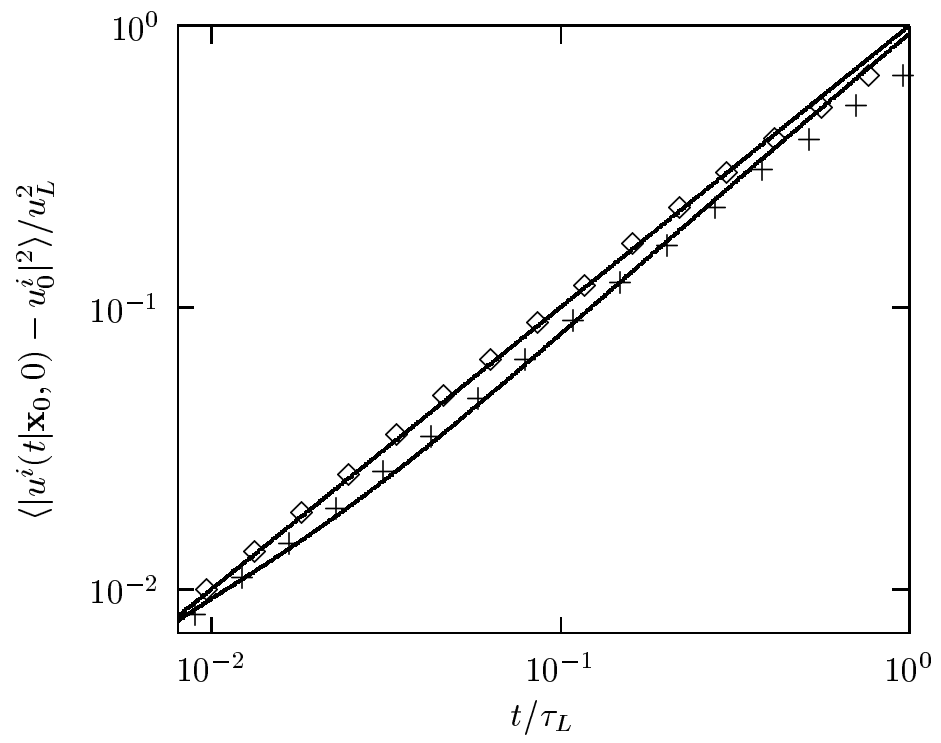

Figure 7: Negative renormalization of $C_{0}$ for small values of $\tau_{S} / \tau_{L}$; $\diamond$ : fluid particle, fitted by Eqn. (14) with $C_{0}=8$ (for $C_{K}=2$ ). $+: \tau_{S} / \tau_{L}=0.0026 u_{G}=0$ fitted by Eqn. (11) with $C_{0}=8$, $a=-0.3$ and $b=1.4$. Using still Eqn. (14), this would correspond to a renormalization $C_{0} \simeq 7$.

be noticed that the renormalization of $C_{0}$ is negative in all the inertia dominated situations, with the exception of the $\tau_{S} \geq 1$ cases, in which the linear spectrum is essentially absent. A negative renormalization of $C_{0}$ corresponds to an inhibition of the time variation for $\mathbf{u}(t)$, which is akin to the increase of the correlation time for $\mathbf{u}(t)$, predicted in [4, 6] in the case of a purely large scale flow. It is not clear, however, how much these phenomena depend upon modelling assumptions.

The crucial aspect seems to be whether the time-statistics is given in an Eulerian frame or along trajectories. An Eulerian time-statistics would suggest a faster fluid velocity variation in a Lagrangian frame 25, which is the physical motivation of the results obtained in [4, 6]. Following the same argument, a time-statistics given along trajectories would lead to the fixed particles feeling the fastest velocity variation. Analytical calculations for random velocity fields with short 
correlation time and Lagrangian time statistics, however, confirm the numerical result of slower velocity variations along particle trajectories 26].

\section{Application to Lagrangian transport modelling}

The analysis carried on so far indicates that the strongest effect of particle inertia on velocity self-correlation is an enhancement of fluctuations at time-scales below $\tau_{S}$ and $\tau_{G}$. Contributions at long time-scales, associated with renormalization of the constant $C_{0}$, remain present even for very light particles, but the correction is small compared to the uncertainties in the value of this constant, even in the case of fluid trajectories. Hence, for time scales above $\tau_{S}$ and $\tau_{G}$, it is acceptable to set $\mathbf{u} \simeq \mathbf{u}^{\mathrm{F}}$ and to model the fluid velocity $\mathbf{u}$ sampled by the heavy particle, with the same Langevin equation used for $\mathbf{u}^{\mathrm{F}}$, i.e. the second of Eqn. (2). Being able to model the difference $\mathbf{u}-\mathbf{u}^{\mathrm{F}}$ becomes necessary, however, when $\tau_{S, G} \sim \tau_{L}$ and trajectory properties at time-scales below $\tau_{S}$ and $\tau_{G}$ become important.

In a Markovian approach like that of [9, 10], the equation for $\mathbf{u}$ would be obtained from the one for $\mathbf{u}^{\mathrm{F}}$, by adding, at discrete times $t_{n}=n \Delta, \Delta<\tau_{S, G}$, uncorrelated increments $\Delta \mathbf{u}$ extracted from a PDF (probability distribution function) in the form $P\left(\Delta \mathbf{u} \mid \Delta \mathbf{x}, \mathbf{u}^{\mathrm{F}}\right)$, with $\Delta \mathbf{x} \sim(\mathbf{v}-\mathbf{u}) \Delta$ the trajectory separation in the time $\Delta$. The shift $\Delta \mathbf{u}$ will have a mean part $\Delta \overline{\mathbf{u}}\left(\mathbf{u}^{\mathrm{F}}\right)$, which is zero when $\mathbf{u}^{\mathrm{F}}=0$ and a fluctuation $\Delta \tilde{\mathbf{u}}: \Delta \tilde{u} \sim u_{\Delta x}$ The cumulative effect of these increments would be felt also at time-scales above $\tau_{S}$ and $\tau_{G}$, in the form of additional noise and drift terms in the equation for $\mathbf{u}$. In particular, the amplitude of the noise correction is estimated from $|\mathbf{u}-\mathbf{v}| \sim u_{S, G}$ and Eqns. $(3,8,12)$ :

$$
\Delta^{-1}(\Delta \tilde{u})^{2} \sim \bar{\epsilon}\left(\tau_{S, G} / \Delta\right)^{\frac{1}{3}}
$$

which is an unphysical result: this amplitude remains finite also in the fluid particle limit $\tau_{S, G} / \tau_{L} \rightarrow$ 0 . The most the drift term can do to improve upon the situation, is to make sure that $\left\langle u^{2}\right\rangle=u_{L}^{2}$ and that the only corrections be in the value of the constant $C_{0}$ and the Lagrangian correlation time $\tau_{L}$ seen by the solid particle. Neglecting the drift, which amounts to extracting the velocity increments from a PDF not conditioned to $\mathbf{u}^{\mathrm{F}}$, would lead to $\left\langle u^{2}\right\rangle>u_{L}^{2}$; this would be equivalent to say that solid particles privilege higher velocity regions of the flow, independently of the value of $\tau_{S, G}$. Going back to the discussion preceeding Eqn. (15), neglecting the conditioning on $\mathbf{u}^{\mathrm{F}}$ would mean that any correlation among velocity increments along fluid trajectories (longitudinal) and from trajectory separation (transverse), is disregarded. (It is to be mentioned that, contrary to the prediction of $[$, 的, the renormalization of the turbulent correlation time, necessary to the fluid 
velocity amplitude constant, leads to a decrease rather than to an increment of $\tau_{L}$ ). Notice that approximating spatial correlations with exponentials [10, 13] is not sufficient to cure this problem; the only gain is eliminating the spurious noise dependence on the discretization $\Delta$. In fact, if we neglected Kolmogorov scaling of space correlations and approximated $\Delta \tilde{u} \sim u_{A}^{2} l / A$, we would still have $\Delta^{-1}(\Delta \tilde{u})^{2} \sim u_{A}^{2} / \tau_{A} \sim \bar{\epsilon}, A=S, G$, which remains finite when $\tau_{S, G} \rightarrow 0$ [compare with Eqn. (20)]. In other words, anomalous diffusion is not an essential ingredient; memory effects are.

Eliminating cumulative effects of the increments in $\mathbf{u}$ leads automatically to the introduction of memory and to a non-Markovian model. A simple Gaussian model with these properties is obtained proceeding in the same philosophy of section III: the velocity $\mathbf{u}$ is modelled by a superposition of solutions of stochastic linear equations, each associated with a spatial scale of the turbulent flow. For homogeneous isotropic turbulence (summation over repeated vectorial indices is understood):

$$
\left\{\begin{array}{l}
u_{i}(t)=\sum_{n} \bar{u}_{i}^{n}(t) ; \\
\frac{\mathrm{d} \bar{u}_{i}^{n}(t)}{\mathrm{d} t}-\bar{\tau}_{n}^{-1} A_{i}^{n}\left(\overline{\mathbf{u}}^{n}\right)=\left(\frac{c_{n} \bar{\epsilon} \tau_{L}}{\bar{\tau}_{n}}\right)^{\frac{1}{2}} \xi_{i}(t) \\
\left\langle\xi_{i}(t) \xi_{j}(0)\right\rangle=\delta_{i j} \bar{\tau}_{n}^{-1} \Xi\left(t / \bar{\tau}_{n}\right) ; \quad \int \mathrm{d} x \Xi(x)=1
\end{array}\right.
$$

where $A_{i}^{n}=-M_{i j}^{n} \bar{u}_{j}^{n}, \bar{\tau}_{n}$ and $M_{i j}^{n}$ are function of $\mathbf{u}-\mathbf{v}, c_{n} \simeq 2^{-\frac{n+2}{2}} C_{0} / 2$, and for $|\mathbf{u}-\mathbf{v}|=0$ : $\bar{\tau}_{n}=2^{-n} \bar{\tau}_{0}$. The noise $\xi_{i}(t)$ must have finite correlation time in order to avoid that the dynamics of $\mathbf{u}$ at a given time-scale $\tau$ receive contribution by shells with $\bar{\tau}_{n} \gg \tau$ (this is implemented automatically in a numerical code, using timesteps proportional to $\bar{\tau}_{n}$, in the different shells). With these prescriptions, for $|\mathbf{u}-\mathbf{v}|=0, \overline{\mathbf{u}}^{n}$ will fluctuate on time scale $\bar{\tau}_{n}$ with amplitude $\left\langle\left|\overline{\mathbf{u}}^{n}\right|^{2}\right\rangle=2^{-n}\left\langle\left|\overline{\mathbf{u}}^{0}\right|^{2}\right\rangle \propto \bar{\tau}_{n}$, and for $t \ll \tau_{L}$ Eqn. (15) will be satisfied.

The variable $\overline{\mathbf{u}}^{n}(t)$ in Eqn. (21) represents the contribution to the Lagrangian velocity component $\mathbf{u}(t)$ from vortices of size $l(n)$ such that $\tau_{l(n)} \sim \bar{\tau}_{n}$; hence $l(n) / L \sim 2^{-\frac{3 n}{2}}$ and then also $\left\langle\left|\overline{\mathbf{u}}^{n}\right|^{2}\right\rangle \sim u_{l(n)}^{2} \sim u_{L}(l(n) / L)^{\frac{2}{3}}$. If $|\mathbf{u}-\mathbf{v}| \gg u_{l(n)}, \overline{\mathbf{u}}^{n}$ is the frozen velocity field of the vortex of size $l(n)$. We have therefore:

$$
\frac{\bar{\tau}_{n}}{\bar{\tau}_{n}(0)}= \begin{cases}1 & |\mathbf{u}-\mathbf{v}| \ll u_{l(n)} \\ \gamma u_{l(n)} /|\mathbf{u}-\mathbf{v}| & |\mathbf{u}-\mathbf{v}| \gg u_{l(n)}\end{cases}
$$

where $\bar{\tau}_{n}(0)$ indicates the value of $\bar{\tau}_{n}$ for $|\mathbf{u}-\mathbf{v}|=0$ and $\gamma$ is an $O(1)$ constant. For $\mid \mathbf{u}-$ $\mathbf{v} \mid \gg u_{l(n)},\left\langle u_{i}^{n}(t) u_{j}^{n}(0)\right\rangle$ is just the space correlation function at separation $(\mathbf{v}-\mathbf{u}) t$ of the flow field produced by vortices at scale $l(n)$. Incompressibility fixes then its tensorial structure [8]: $\left\langle u_{i}^{n}(t) u_{j}^{n}(0)\right\rangle=f\left(t / \bar{\tau}_{n}\right) L_{i j}+g\left(t / \bar{\tau}_{n}\right)\left(\delta_{i j}-L_{i j}\right)$ with $L_{i j}=\frac{(\mathbf{u}-\mathbf{v})_{i}(\mathbf{u}-\mathbf{v})_{j}}{|\mathbf{u}-\mathbf{v}|^{2}}$ and in three dimensions: $g(x)=f(x)+\frac{x}{2} f^{\prime}(x)$. Using Eqn. (13) also for $|\mathbf{u}-\mathbf{v}| \gg u_{l(n)}$ is equivalent to assuming exponential space correlations, and from $f(x)=\exp (-x)$ we find $g(x)=\left(1-\frac{x}{2}\right) \exp (x) \simeq \exp \left(-\frac{3}{2} x\right)$. In the 
opposite limit $|\mathbf{u}-\mathbf{v}| / u_{l(n)} \rightarrow 0$, isotropy imposes $\left\langle u_{i}^{n}(t) u_{j}^{n}(0)\right\rangle=f\left(t / \bar{\tau}_{n}\right) \delta_{i j}$. These conditions fix the form of the tensor $M_{i j}^{n}$ in the two opposite regimes:

$$
M_{i j}^{n}= \begin{cases}\delta_{i j} & |\mathbf{u}-\mathbf{v}| \ll u_{l(n)} \\ \frac{3}{2} \delta_{i j}-\frac{1}{2} L_{i j} & |\mathbf{u}-\mathbf{v}| \gg u_{l(n)}\end{cases}
$$

The model described by Eqns. (21-23) is reminiscent of a continuous time random walk 27, in that the non-Markovian nature of the process originates from varying the correlation times of the various contribution to $\mathbf{u}(t)$, rather than their amplitudes, which remain invariant.

The self-diffusion properties of the solution to Eqns. (21-23), limited to one dimension, are compared in Figs. 8 and 9, with the synthetic turbulence results of section III. The time $\tau_{L}$ and the noise amplitude $C_{0} \bar{\epsilon}$ in Eqn. (15) have been selected to fit the synthetic turbulence result for $\tau_{S}=\tau_{G}=0$ and the expression $\bar{\tau}_{n} / \bar{\tau}_{n}(0)=\left(1+0.3|\mathbf{u}-\mathbf{v}| / u_{l(n)}\right)^{-1}$ has been used for the correlation times. When $\bar{\tau}_{n}<\bar{\tau}_{N}(0)$, with $N$ the fastest shell, mode $n$ was disregarded and the fastest mode with correlation time above $2^{-N} \tau_{L}$ was renormalized to account for the energy of the lost shell.

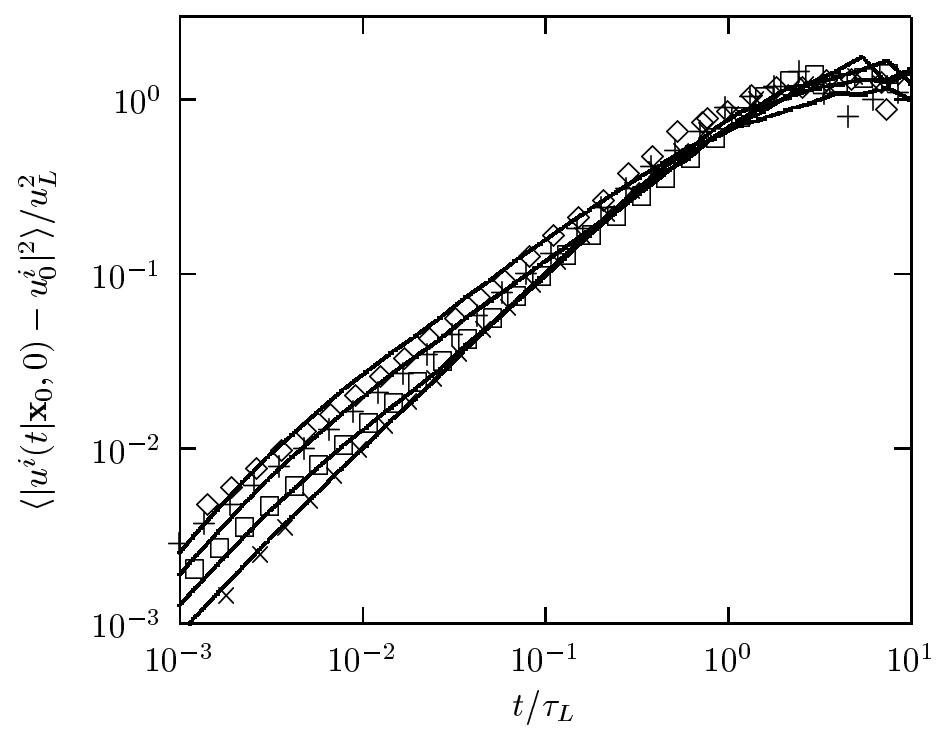

Figure 8: Comparison of solution to 1-dimensional version of Eqns. (21-23) (continuous lines) and synthetic turbulence results for velocity self-diffusion. ' $\diamond^{\prime} \tau_{S} / \tau_{L}=2.6 .{ }^{\prime}+{ }^{\prime} \tau_{S} / \tau_{L}=0.26 .{ }^{\prime} \square^{\prime}$ $\tau_{S} / \tau_{L}=0.026 .{ }^{\prime} \times{ }^{\prime} \tau_{S}=0$. Equation $(21)$ has been solved with 13 modes and $\bar{\tau}_{0}(0) \simeq 0.35 \tau_{L}$.

The model just described allows to keep into account the Kolmogorov-like nature of the small scale spatial structure of high Reynolds number turbulence. It is easy to see this. From the discussion in Section II, the typical particle-fluid relative velocity is $|\mathbf{u}-\mathbf{v}| \sim \max \left(u_{S}, u_{G}\right)$. The 


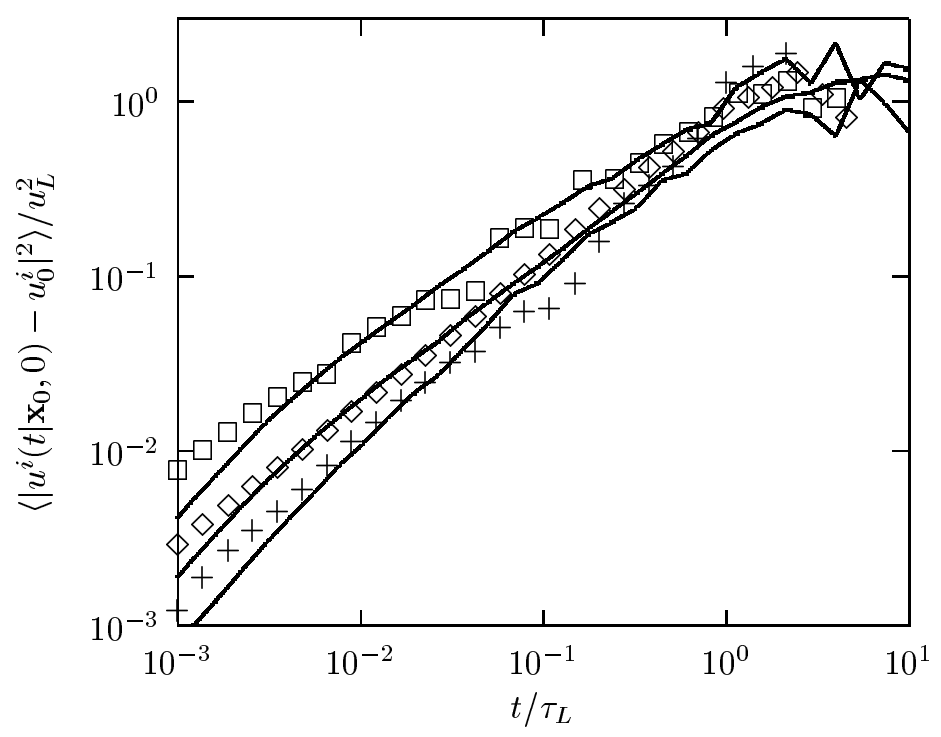

Figure 9: Another comparison of solutions of Eqns. (21-23) (continuous line) and synthetic turbulence results. Here, self-diffusion is conditioned to different values of the ratio $\langle|\mathbf{u}-\mathbf{v}|\rangle^{-1}|\mathbf{u}-\mathbf{v}|$, for fixed $\tau_{S} / \tau_{L}=0.26, u_{G}=0:^{\prime}+^{\prime}: 2<\langle|\mathbf{u}-\mathbf{v}|\rangle^{-1}|\mathbf{u}-\mathbf{v}| .^{\prime} \square^{\prime}:\langle|\mathbf{u}-\mathbf{v}|\rangle^{-1}|\mathbf{u}-\mathbf{v}|<0.12 .^{\prime} \diamond^{\prime}$ : unconditioned. Equation (21) has been solved using the same parameters of Fig. 8.

fluid velocity increment along a solid particle trajectory $\left\langle|\mathbf{u}(t)-\mathbf{u}(0)|^{2}\right\rangle$ will be dominated by that shell for which $\bar{\tau}_{n}\left(u_{S, G} / u_{l(n)}\right)=t$ and $t=\tau_{S, G}$ will give the cross-over from the situation in which the corresponding $\bar{\tau}_{n}$ obeys the first or the second of Eqn. (22). From Eqn. (22), and the scaling for the shell characteristic time and velocity: $\bar{\tau}_{n}(0)=\bar{\tau}_{0} 2^{-n},\left\langle\left|\overline{\mathbf{u}}^{n}\right|^{2}\right\rangle \sim u_{L}^{2} 2^{-n-1}$, we thus obtain:

$$
\bar{u}_{n} / u_{S, G} \sim \begin{cases}\left(t / \tau_{S, G}\right)^{\frac{1}{2}} & t \gg \tau_{S, G} \\ \left(t / \tau_{S, G}\right)^{\frac{1}{3}} & t \ll \tau_{S, G}\end{cases}
$$

At short times, the fluid velocity self-diffusion will have the $t^{\frac{1}{3}}$ scaling, which comes from the spatial profile of the small vortices being seen as frozen by the crossing solid particle. Conversely, at long times, we will find the usual $t^{\frac{1}{2}}$ normal scaling of the Lagrangian time structure function [see Eqn. (4)], which is obtained disregarding trajectory separation, and equating $t$ with the eddy turn-over time.

The problem of Markovian models [9, 10], discussed at the beginning of this section, disappears in the present approach. Trajectory crossing effects, in the limit $\tau_{S, G} \rightarrow 0$, are confined to shells with $n \rightarrow \infty$, and their contribution to $T_{L}$ disappears in the limit (as it should). In this respect, this model is closer to the ones by [3, 28], in which the effect of particle inertia is accounted for by correlation time renormalization, rather than modelling directly the change of fluid velocity from trajectory crossing. Of course, the intent of modelling the turbulent small scales, adopting either 
the Markovian approach of [9, 10], or the non-Markovian approach used here and in 14, 15, was completely absent in [3, 28].

In basically all existing theories of heavy particle dispersion, the Kolmogorov structure of small scale fluctuations is disregarded. Typically, an exponential [10, 13, 28] or Gaussian [4, 5 profile for the space correlations is adopted. In some approaches [4, 5], also the Lagrangian nature at short time separations, of the time correlations, is neglected.

After first submission of the present work, this author became aware of the paper by Reynolds and Cohen 29], in which a fractional Brownian motion algorithm was used to generate the anomalous scaling behaviors resulting from trajectory crossing. The advantage of the present approach is the predictive ability for the scaling, whose physical mechanis is built in the algorithm by means of the shell superposition technique. It is interesting to analyze the prediction of the present model on the test case considered in [29] based on the Snyder and Lumley experimental data [30]. Figure 10 illustrates a power law fit for the fluid velocity self diffusion along a solid particle trajectory simulated with Eqns. (21-23), with particle parameters corresponding to the "glass", "corn pollen" and "hollow glass" particle case considered in [30]. The same form for $\bar{\tau}_{n}$ used before:

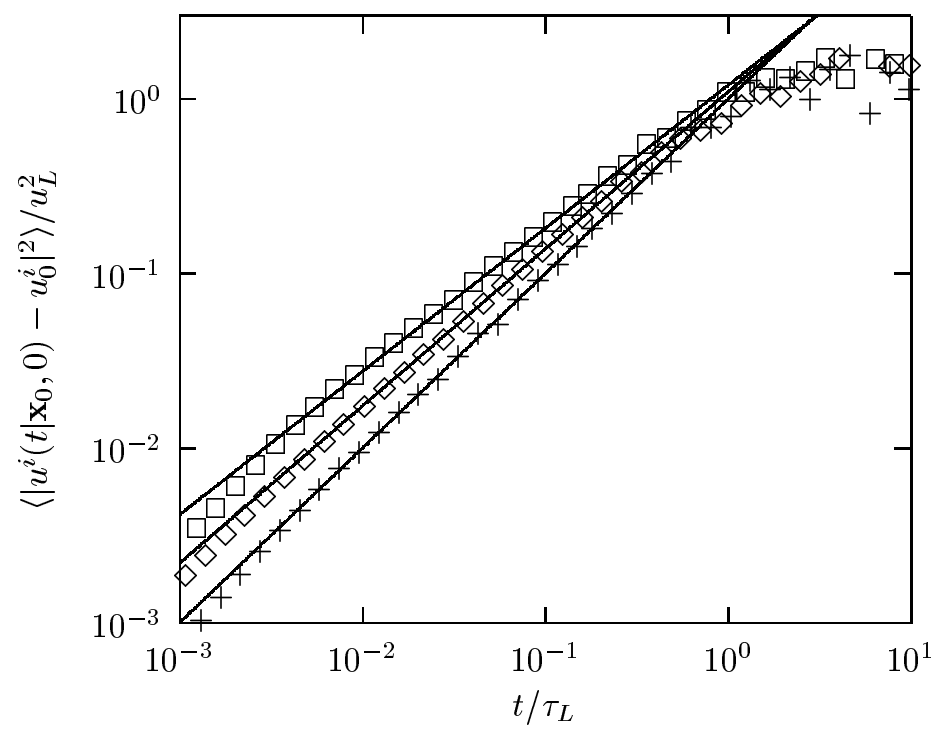

Figure 10: Fluid velocity self-diffusion predicted by Eqns. (21-23) in three test cases; $\square$ (glass): $u_{G} / u_{L} \simeq 1.76, \tau_{S} / \tau_{L} \simeq 0.26 ; \diamond$ (corn pollen) $u_{G} / u_{L} \simeq 0.7, \tau_{S} / \tau_{L} \simeq 0.1 ;+$ (hollow glass) $u_{G} / u_{L} \simeq 0.052, \tau_{S} / \tau_{L} \simeq 0.01$. The continuous line illustrate the power law fit by $t^{2 H} ;$ in the three cases: $H \simeq 0.41 ; H=0.45$ and $H \simeq 0.5$.

$\bar{\tau}_{n} / \bar{\tau}_{n}(0)=\left(1+0.3|\mathbf{u}-\mathbf{v}| / u_{l(n)}\right)^{-1}$, leading to the results of Figs. 8 and 9 , continued to be used. In the three cases the fit gives for the exponent $H:\left\langle\left|\mathbf{u}\left(t \mid \mathbf{x}_{0}, 0\right)-\mathbf{u}_{0}\right|^{2}\right\rangle \sim t^{2 H}, H \simeq 0.41, H \simeq 0.45$ 
and $H \simeq 0.5$. This agrees well with the results in [29] with the exception of the hollow glass case, in which a behavior closer to that of a fluid parcel is predicted here.

A shortcome of the present model is clearly that the exact form of the shell time-scales must be provided from the outside, since Eqn. (22) is only valid asymptotically. Among the other things, knowing the exact form of $\bar{\tau}_{n} / \bar{\tau}_{n}(0)$ for $|\mathbf{u}-\mathbf{v}| / u_{l(n)} \lesssim 1$ would be necessary, at least for small $n$, to determine the fluid velocity correlation sampled by the solid particle. This shortcome is common also to other models [2, 28], in which the renormalization of the correlation time by inertia must be introduced as an external ingredient. It is to be stressed, however, that the inertia renormalization of the correlation time belongs, together with the determination of the constant $C_{0}[12$ and of the relative size of Eulerian and Lagrangian correlation times [21], to a class of problems which are havily influenced by the turbulent large scales. It is thus to be expected that this parameter be dependent on the nature of the flow and it be necessary to provide it from the outside.

It is possible to extend the Lagrangian modelling approach illustrated in this paper to nonGaussian inhomogeneous turbulence situations, imposing, in the fluid particle limit $|\mathbf{u}-\mathbf{v}|=0$, individually on each shell, the well-mixedness condition [11]. It must be stressed that the well mixedness condition can be imposed only in the fluid particle limit $\mathbf{v}=\mathbf{u}$. Apart of all the problems connected with ergodicity of solid particle trajectories, the particle concentration profile in the inhomogeneous turbulence flow is unknown and, in fact, is typically one of the desired outputs of the model.

Basically, one has a condition on $\bar{\epsilon}(\mathbf{x}, t)$ from the integral time scale:

$$
\bar{\epsilon}(\mathbf{x}, t)=\frac{u_{L}^{2}(\mathbf{x}, t)}{C_{0} \tau_{L}(\mathbf{x}, t)}
$$

plus a condition for each of the (tensor) moments which are experimentally available:

$$
\sum_{n}\left\langle\left[\overline{\mathbf{u}}^{n}\right]^{p}\right\rangle(\mathbf{x}, t)=\left\langle\mathbf{u}^{p}\right\rangle(\mathbf{x}, t)
$$

with Kolmogorov scaling giving, for $p=2:\left\langle\bar{u}_{n}^{2}\right\rangle(\mathbf{x}, t)=2^{-n-1} u_{L}^{2}(\mathbf{x}, t)$. The choice of the contribution from each shell to the non-Gaussian part of the statistics remains arbitrary, conditioned to statistical realizability of the moments for the $\bar{u}_{n}$.

A possibility could be to partition the contribution equally among different shells: $\left\langle\left[\overline{\mathbf{u}}^{n}\right]^{p}\right\rangle_{c}(\mathbf{x}, t)=$ $2^{-\frac{p(n+1)}{2}}\left\langle[\mathbf{u}]^{p}\right\rangle_{c}(\mathbf{x}, t)$, with subscript $c$ staying for cumulant. A choice which is more consistent with the standard practice in stochastic Lagrangian models, to let the non-Gaussianity be generated by a non-linearity in the drift term, considering it therefore to be a purely large scale 
feature of the flow, would be to concentrate all the non-Gaussianity production in the first shell: $\left\langle\left|\overline{\mathbf{u}}^{n}\right|^{p}\right\rangle_{c}(\mathbf{x}, t)=\left\langle u^{p}\right\rangle_{c}(\mathbf{x}, t) \delta_{1, n}$.

Once the PDF's $P_{n}\left(\overline{\mathbf{u}}^{n}, \mathbf{x}, t\right)$ for the various shells are obtained from the cumulants through some reconstruction technique [31], the Fokker-Planck equation for the second of Eqn. (21) can be inverted to determine the drift terms $A_{i}^{n}$ from $\bar{\epsilon}$ and $P_{n}$; considering for simplicity the case of stationary turbulence:

$$
\partial_{\bar{u}_{i}^{n}}\left(A_{i}^{n} P_{n}\right)=-\bar{\tau}_{n}(0) \bar{u}_{i}^{n} \partial_{i} P_{n}+\frac{c_{n} \bar{\epsilon} \tau_{L}}{2} \partial_{\bar{u}_{i}^{n}} \partial_{\bar{u}_{i}^{n}} P_{n}
$$

As it is well known (see e.g. 31]), in inhomogeneous turbulence situations and for $\left\langle\overline{\mathbf{u}}^{n}\right\rangle=0$, Eqn. (25) leads to a constant contribution to the drift, which counterbalances the $\operatorname{drift} A_{i}^{n}$ from the gradient term $\bar{u}_{i}^{n} \partial_{i} P_{n}$ and enforces zero mean for $\overline{\mathbf{u}}^{n}$.

Notice that this balance disappears in the case of the solid particle flow, in which case the particle velocity will be characterized by a non-zero turboforetic mean drift. The multivariate PDF $P\left(\left\{\overline{\mathbf{u}}^{n}\right\}, \mathbf{v}, \mathbf{x}\right)$ corresponding to a uniform particle concentration will obey the Fokker-Planck equation:

$$
v_{i} \partial_{i} P-\partial_{v_{i}}\left(\frac{v_{i}-u_{i}-u_{G i}}{\tau_{S}} P\right)+\sum_{n} \partial_{\bar{u}_{i}^{n}}\left(\bar{\tau}_{n}^{-1} A_{i}^{n} P\right)=\sum_{n} \frac{c_{n} \bar{\epsilon} \tau_{L}}{2} \partial_{\bar{u}_{i}^{n}} \partial_{\bar{u}_{i}^{n}}\left(P / \bar{\tau}_{n}\right)
$$

from which, for small $\tau_{S} / \tau_{L}$ (and then $u_{S} \ll \mathbf{u}_{L}$ ), the drift can easily be calculated. Multiplying Eqn. (26) by $v_{i}$, integrating over $\mathbf{v}$ and $\left\{\overline{\mathbf{u}}^{n}\right\}$, and using $\left\langle v_{i} v_{j}\right\rangle=\left\langle u_{i} u_{j}\right\rangle+O\left(u_{S}^{2}\right) \simeq\left\langle u_{i} u_{j}\right\rangle$, we obtain in fact:

$$
\left\langle v_{i}\right\rangle=-\tau_{S} \partial_{j}\left\langle u_{i} u_{j}\right\rangle
$$

Thanks to the well-mixedness condition, however, this turboforetic drift is free of spurious contributions from unproper Lagrangian modelling of the fluid limit case.

It must be said that, contrary to what happens (at least in part) in a model like [1], the time structure of the individual trajectories is here physically relevant. This because of the coupling between $\mathbf{u}$ and $\mathbf{v}$ through Eqn. (1). Trying to base an extension of the present model to nonhomogeneous non-Gaussian turbulence, on a statistical constraint like well-mixedness, may thus be of limited value. It must also be said that the ergodic property is not satisfied in general by heavy particles. (In the case of fluid elements, the ergodic property is always assumed, implicitly, as a working hypothesis). It is not clear, therefore, whether it is appropriate to hypothesize invariance even only of the amplitude for $\mathbf{u}(t)$, when passing from $|\mathbf{u}-\mathbf{v}|=0$ to the heavy particle case $|\mathbf{u}-\mathbf{v}|>0[26$. 


\section{Conclusions}

The analysis carried out in this paper provides a picture of the behavior of heavy particles in turbulent flows, and, in particular, a prediction for the scaling properties of the fluid velocity sampled by the particles, in function of the flow and particle properties. The predictions based on scaling analysis were confirmed by synthetic turbulence simulations and allowed the derivation of a Lagrangian transport model taking into account the non-Markovian nature of the fluid velocity along the heavy particle trajectory.

It is important to understand that this non-Markovian nature cannot be overlooked without getting into contradictions. For instance, it would be rather natural, in a model like [9, 10], to hypothesize a normal diffusion behavior for $\mathbf{u}^{\mathrm{F}}$ at high Reynolds numbers, and to study pairs of particle-fluid trajectories of duration $\Delta \leq \tau_{S}$, in which the approximation $\mathbf{u} \simeq \mathbf{u}^{\mathrm{F}}$ is used. But, as discussed at the beginning of Section IV, if one neglects correlations between the velocity increments $\Delta \mathbf{u}=\mathbf{u}-\mathbf{u}^{\mathrm{F}}$ at the discrete times $t_{n}=n \Delta$, modifications in the time correlations sampled by the solid particle will appear up to time-scale $\tau_{L}$ and remain present in the fluid particle limit $\tau_{S, G} \rightarrow 0$, which is unphysical. Even worse, if the PDF $P\left(\Delta \mathbf{u} \mid \mathbf{u}^{\mathrm{F}}\right)$, from which $\Delta \mathbf{u}$ is

extracted, is not chosen carefully enough, and the condition on $\mathbf{u}^{\mathrm{F}}$ is disregarded by choosing say $P(\Delta \mathbf{u})=P\left(\mathbf{u}^{\mathrm{E}}(\mathbf{x}+\Delta \mathbf{x}, t)-\mathbf{u}^{\mathrm{E}}(\mathbf{x}, t)\right)$, one will find also $\left\langle u_{i}^{2}\right\rangle \neq u_{L}^{2}$.

The scaling analysis suggests a picture of transients between different spectral slopes at different time-scales, in which the controlling parameters are $\tau_{S}$ and $u_{S}\left(\tau_{G}\right.$ and $u_{G}$ in the case of strong external forces), which are the time and velocity scales for $\mathbf{u}-\mathbf{v}$. Both synthetic turbulence simulations and the runs from the Lagrangian model, however, indicated that cross-over behaviors rather than pure scaling dominate even for very high Reynolds numbers.

In the last section, some ideas for inclusion of non-Markovian effects in a Lagrangian stochastic model for solid particle transport have been illustrated. In this way, the Kolmogorov nature of the turbulent small scales and the Lagrangian character of time correlations is fully taken into account. The suggested approach is based on the use of a superposition of Markovian processes, which will be slower than a simpler Markovian model by a factor of the order of the number of terms in the superposition. (In our case, with 12 shells, the ratio was approximately 8). A similar approach has been recently used to model turbulence intermittency [32].

An alternative approach to generate the sub-diffusive behavior for the velocity, at time-scales below $\tau_{S, G}$, could have been to resort to a continuous time random walk 27], or, equivalently, 
to Lèvy walks [33]. This would have been lighter from the computational point of view, but the control on the form of the PDF for the velocity and the velocity difference would have been much more difficult. The control on the shape of the correlations for long times, would have been difficult as well. Notice that, in the present approach, recirculation phenomena and correlations modelled on Frenkiel functions [8, 9] could easily have been taken into account in Eqn. (21) by the use of complex relaxation times.

Compared to the recent model by Reynolds and Cohen [29], the present approach provides in self-consistent way the scaling exponent for the fluid velocity self-diffusion. This both in the case of dominant inertia and dominant external forces and with no special cost in terms of simplicity and of the possibility of imposing a well mixedness condition in inhomogeneous non-Gaussian turbulence situations.

To obtain a Lagrangian model of practical use, a precise form for the dependence of the shell correlation time $\bar{\tau}_{n}$ on $\mathbf{u}-\mathbf{v}$ should be provided. The two-dimensional synthetic turbulence simulations, which have been carried out, can at most confirm scaling behaviors and perhaps give the sign of the inertia produced correction to the correlation time [see Eqn. (19)]. The form of the correlation times $\bar{\tau}_{n}$ could probably be obtained only by DNS of the full Navier-Stokes equation in the proper tree-dimensional flow conditions.

However, although other such attempts to take into account the Kolmogorov structure of the turbulent small scales, were already carried on [14, 15], it is still not clear how relevant these aspects are to the derivation of a dispersion model of practical use. Probably, the main interest remains conceptual. Considering the reduced information on the turbulent flows available in practical situations, and the fact that dispersion is governed by the local large scales anyway, a minimal implementation of the model could disregard the small scales altogether and it could be sufficient to keep only one shell in Eqn. (21). In such a hyper-simplified version, the present model would become equivalent to [28, with a generalized form of the Lagrangian correlation profile for $\tau_{S}=0$ to allow enforcement of the well mixedness condition.

Aknowledgements: I wish to thank Paolo Paradisi for interesting discussion and for his contribution to the initial part of this research, and Francesco Tampieri for insightful comments.

\section{A. Appendix}

In this appendix, a closer look is given to the synthetic turbulence model described in section 
III. In this approach, the effect of a homogeneous turbulent flow is modelled by a velocity field localized around the particles that one is tracking. This limits the evaluation of spatial objects like e.g. correlation functions, to the case in which the correlation points coincide with the particles. This approach, has been used to analyze the process of relative diffusion (see Figs. 2 and 3), but, as already discussed, is plagued by difficulties. The space structure functions can be obtained in a simpler way from the assumption that the local field around the particle is representative of the whole flow. The Eulerian velocity field is in the form:

$$
\mathbf{u}^{\mathrm{E}}(\mathbf{x}, t)=\sum_{i} \overline{\mathbf{u}}_{i}(\mathbf{x}, t)
$$

where $i$ labels the vortex. The contribution from vortex $i$ is

$$
\overline{\mathbf{u}}_{i}(\mathbf{x}, t)=\alpha r_{i}^{\frac{1}{3}} W_{i} \mathbf{R}\left(\frac{\mathbf{x}-\overline{\mathbf{x}}_{i}(t)}{r_{i}}\right) T\left(\frac{t-\bar{t}_{i}}{\bar{\tau}_{r_{i}}}\right)
$$

where $W_{i}$ are uncorrelated zero mean and unitary variance random variables, $\alpha$ is a constant and $\overline{\mathbf{x}}_{i}(t)$ and $\bar{t}_{i}$ indicate respectively the vortex center position at time $t$ and the time of "birth" of vortex $i$. We have considered vortices with compact support in space and time; precisely:

$$
\left\{\begin{array}{l}
\mathbf{R}(\mathbf{x})=\mathbf{e}_{3} \times \mathbf{x} H(1-x) \\
T(t)=H(t(1-t))
\end{array}\right.
$$

where $H(x)=x \theta(x)$ with $\theta(x)$ the Heaviside step function. The lifetime of the vortex has been taken as a deterministic function of the radius: $\bar{\tau}_{r}=\beta r^{\frac{2}{3}}$.

Since the $W_{i}$ are uncorrelated, the two-point correlation reduces to a sum of contributions from the individual vortices:

$$
\langle\mathbf{u}(\mathbf{x}, t) \mathbf{u}(0,0)\rangle=\alpha^{2} \int \mathrm{d} \log r \int \mathrm{d} \bar{t} \int \mathrm{d}^{2} \bar{x} N(r) r^{\frac{2}{3}} T\left(\frac{t-\bar{t}}{\bar{\tau}_{r}}\right) T\left(\frac{-\bar{t}}{\bar{\tau}_{r}}\right) \mathbf{R}\left(\frac{\mathbf{x}-\overline{\mathbf{x}}}{r}\right) \mathbf{R}\left(-\frac{\mathbf{x}}{r}\right)
$$

If any point feels on the average the effect of a fixed number of vortices per shell, the vortex density $N(r)$ can be written in the form

$$
N(r)=\bar{N}\left(\pi r^{2} \bar{\tau}_{r}\right)^{-1}
$$

with $\bar{N}$ a constant, which is exactly $1 / \log 2$ in our case of one vortex per octave contributing to velocity at a given time and position.

Substituting Eqns. (A3) and (A5) into Eqn. (A5), one can find the expression for the longitudinal strucure function $S_{2}(l)=\left\langle\left(u_{1}\left(l \mathbf{e}_{1}, 0\right)-u_{1}(0,0)\right)^{2}\right\rangle$ :

$$
S_{2}(l)=\frac{\alpha^{2}}{15 \pi \ln 2} \int \frac{\mathrm{d} r}{r^{\frac{1}{3}}} \int \mathrm{d}^{2} \bar{x} R_{1}(\overline{\mathbf{x}})\left[R_{1}\left(\overline{\mathbf{x}}+(l / r) \mathbf{e}_{1}\right)-R_{1}(\overline{\mathbf{x}})\right]
$$


Integrating numerically, one finds: $S_{2}(l) \simeq 0.0039 \alpha^{2} l^{\frac{2}{3}}$, i.e. in terms of Kolmogorov constant and mean dissipation:

$$
\alpha \simeq 16 C_{K}^{\frac{1}{2}} \bar{\epsilon}^{\frac{1}{3}}
$$

In Fig. 11 below is illustrated the structure function for an 18-shell synthetic tubulence field. All

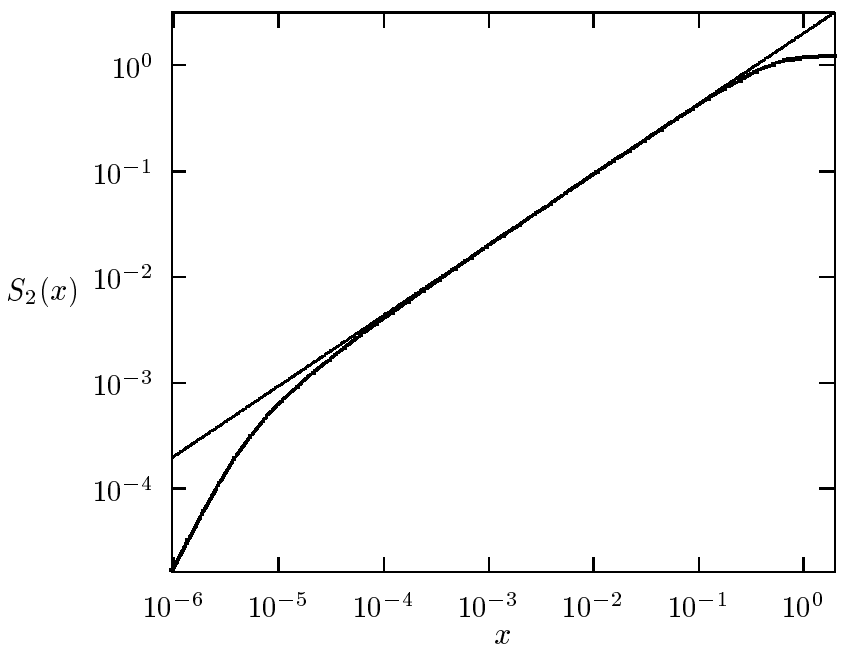

Figure 11: Space structure function from an 18-shell synthetic turbulence field with $\alpha=22.627$, $L=1$ and fit by a Kolmogorov spectrum $S_{2}(x)=2 x^{\frac{2}{3}}$.

simulations have been carried out with $\beta \simeq 8.3 / \alpha$, corresponding to an eddy life of the same order of the eddy turn-over time. (Large and small values of $\beta$ correspond respectively to long and short vortex lives).

Once we know the coefficients for $x^{-\frac{2}{3}} S_{2}(x)$ and $t^{-1}\left\langle\left|u_{1}(t)-u_{1}(0)\right|^{2}\right\rangle$, from Eqns. (10) and (A7), we can eliminate the dissipation $\bar{\epsilon}$ to find the ratio $C_{0}^{-\frac{3}{2}} C_{k}$. We obtain from the simulations $C_{K}^{-\frac{3}{2}} C_{0} \simeq 2.828$. If we take for the Kolmogorov constant $C_{K} \simeq 2$, we will have $C_{0} \simeq 8$, which is somewhat large. The explanation of this fact may lie in the way a particle deviates from the flow line of a given vortex due to the effect of vortices at other scales in the same region. In such a model, this effect is magnified by the rigid structure of the vortices, and a particle will move out of the one in which it lies at a given time, faster than it would do if the vortex were able to be deformed by the flow. More important, however, is probably the fact the this flow is two-dimensional and it would be interesting therefore to compare it with a closer physical situation like two-dimensional turbulence in the inverse cascade range.

Following the same approach, it is possible to calculate $u_{L}$ and, from Eqns. (3-4) also $L$ and 
$\tau_{L}$. The main parameters of the synthetic turbulent field are listed in Table I below.

\begin{tabular}{|l|l|l|}
\hline$u_{L} \simeq 0.61$ & $T_{L} \simeq 0.07$ & $C_{0}^{-1} \tilde{c} \simeq 0.003$ \\
$L \simeq 0.17$ & $C_{K}^{-\frac{2}{3}} C_{0} \simeq 2.83$ & $\alpha=22.627$ \\
$\tau_{L} \simeq 0.2$ & $C_{0}^{-1} c \simeq 0.29$ & $\beta=0.367$ \\
\hline
\end{tabular}

Table I

The last issue is how the vortices were generated in the synthetic turbulence field. The total number of vortices in shell $n$ was taken equal to the integer part of a variable $\mathcal{N}$, obeying an equation in the form:

$$
\dot{\mathcal{N}}_{n}=-\tau_{n}^{-1}(|\mathbf{u}-\mathbf{v}|) \operatorname{sign}\left(N_{n}-1\right)
$$

where $N_{n}$ is the number of vortices having the particle in their support at the given time and $\tau_{n}(|\mathbf{u}-\mathbf{v}|)$ is the average time a particle travelling across the fluid with relative velocity $\mathbf{v}-\mathbf{u}$ feels the effect of a vortex in shell $n$ :

$$
\tau_{n}(|\mathbf{u}-\mathbf{v}|) \sim 2^{-\frac{2 n}{3}} F\left(2^{-\frac{1}{3}}|\mathbf{u}-\mathbf{v}|\right)
$$

with $F(x) \propto x^{-1}$ for $x$ large. In this way, the number of vortices could be adjusted, in a time of the order of the vortex life, in such a way that the particle felt the effect of only one vortex per shell.

If the number of vortices alive at a given time was less than $\mathcal{N}$, a new one was put in the shell in an appropriate domain around the particle and with an age chosen at random between zero and the vortex lifetime. The domain was an annular region comprised between $r$ and $\gamma r$ with $\gamma>1$, in such a way that the velocity at the particle position did not suffer from discontinuities. In all simulations, we set $\gamma=2.3$; with this choice, it was enough to keep $\mathcal{N} \leq 4$ in all simulations, with the exception of those with $\tau_{S} / \tau_{L}=2.6$ which required $\mathcal{N} \leq 5$. As in other shell models, the main limitation was not the number of degrees of freedom, but the strongly different time scales involved when the number of shells becomes large.

Apart of the potential increase in the number of eddies required in the neighborhood of a particle, and of the conceptual problems associated with the eddy geometry, extension of the present technique to three dimensions is clearly straightforward.

\section{References}

[1] M.I. Yudine, "Physical considerations on heavy particle diffusion," Adv. in Geophys. 6, 185 $(1959)$ 
[2] G.T. Csanady, "Turbulent diffusion of heavy particles in the atmosphere," J. Atmos. Sci. 20, $201(1963)$

[3] B.L. Sawford and F.M. Guest, "Lagrangian statistical simulations of the turbulent motion of heavy particles," Boundary-Layer Meteorol. 54, 147 (1991)

[4] L.M. Pismen and A. Nir, "On the motion of suspended particle in stationary homogeneous turbulence," J. Fluid Mech. 84, 193 (1978)

[5] A. Nir and L.M. Pismen, "The effect of a steady drift on the dispersion of a particle in turbulent fluid," J. Fluid Mech. 94, 369 (1979)

[6] M.W. Reeks, "On the dispersion of small particles suspended in an isotropic turbulent fluid," J. Fluid Mech. 83, 529 (1977)

[7] T.-H. Shih and J.L. Lumley, "Second-order modelling of particle dispersion in a turbulent flow," J. Fluid Mech. 163, 349 (1986)

[8] J.O. Hinze, Turbulence (McGraw-Hill, New York, 1975)

[9] P. Desjonqueres, A. Berlemont and G. Gouesbet, "A Lagrangian approach for the prediction of particle dispersion in turbulent flows," J. Aerosol Sci. 19, 99 (1988)

[10] A. Berlemont, P. Desjonqueres and G. Gouesbet, "Particle Lagrangian simulation in turbulent flows," Int. J. Multiphase Flow 16, 19 (1990)

[11] D.J. Thomson, "Criteria for the selection of stochastic models of particle trajectories in turbulent flows," J. Fluid Mech. 180, 529 (1987)

[12] B.L. Sawford, "Reynolds number effects in Lagrangian stochastic models of turbulent dispersion," Phys. Fluids A3, 1577 (1991)

[13] Q.Q. Lu, J.R. Fontaine and G. Aubertin, "Particle motion in two-dimensional confined turbulent motion," Aerosol Science and Technology 17, 169 (1992)

[14] Y.-P. Shao, "A Lagrangian stochastic model for non-passive particle diffusion in turbulent flows," Math. Comput. Modelling, 21, 31 (1995)

[15] A.M. Reynolds, "On the formulation of Lagrangian stochastic models for heavy particle trajectories," J. Colloid Interface Sci. 232, 260 (2000) 
[16] P. Olla, "Three applications of scaling to inhomogeneous anisotropic turbulence," Phys. Rev. E 57, $2824(1998)$

[17] J.L. Synge and C.C. Lin, "On a statistical model of isotropic turbulence," Trans. R.S.C. sec. III 37, 45 (1943)

[18] M. Abel, L. Biferale, M. Cencini, M. Falcioni, D. Vergni and A. Vulpiani, "Exit-times and $\epsilon$-entropy for dynamical systems, stochastic processes and turbulence," Physica D 147, 12 $(2000)$

[19] G.A. Kallio and M.W. Reeks, "A numerical simulation of particle deposition in turbulent boundary layers," Int. J. Multiphase Flow 15, 433 (1989)

[20] B.Y. Underwood, "Random walk modelling of turbulent impaction to a smooth wall," Int. J. Multiphase Flow 19, 485 (1993)

[21] S.R. Hanna, "Lagrangian and Eulerian time-scale relations in the daytime boundary layer," J. Appl. Met. 20, 242 (1981)

[22] J.C.H. Fung, J.C.R. Hunt, N.A. Malik and R. Perkins, "Kinematic simulation of homogeneous turbulence by unsteady random Fourier modes," J. Fluid Mech. 236, 281 (1992)

[23] S. Ott and J. Mann, "An experimental investigation of the relative diffusion of particle pairs in three-dimensional turbulent flow," J. Fluid Mech. 422, 207 (2000)

[24] G. Boffetta, A. Celani and A. Vulpiani, "Relative dispersion in fully developed turbulence: from Eulerian to Lagrangian in synthetic flows," Phys. Rev. E 60, 6734 (1999)

[25] R.H. Kraichnan, "Relation between Lagrangian and Eulerian correlation times of a turbulent velocity field," Phys. Fluids 7, 142 (1967)

[26] P. Olla, "Particle transport in a random velocity field with Lagrangian statistics," nlin.CD/0201003

[27] E.W. Montroll, "Random walks on lattices," Proc. Symp. Appl. Math. 16, 193 (1964)

[28] L.-P, Wang and D.E. Stock, "Dispersion of heavy particles by turbulent motion," J. Atmos. Sci. 50, 1897 (1993) 
[29] A.M. Reynolds and J.E. Cohen, "Stochastic simulation of heavy-particle trajectories in turbulent flows," Phys. Fluids 14,342 (2002)

[30] W.H. Snyder and J.L. Lumley "Some measurements of particle velocity autocorrelation in a turbulent flow," J. Fluid Mech. 48, 41 (1971)

[31] S. Du, J.D. Wilson and E. Yee, "On the moments approximation method for constructing a Lagrangian stochastic model," Boundary-Layer Meteorol. 40, 273 (1994)

[32] L. Biferale, G. Boffetta, A. Celani, A. Crisanti and A. Vulpiani, "Mimicking a turbulent signal: sequential multiaffine processes," Phys. Rev. E 57, R6261 (1998)

[33] M.F. Schlesinger, B.J. West and J. Klafter, "Lèvy dynamics of enhanced diffusion: application to turbulence," Phys Rev. Lett. 58, 1100 (1987) 